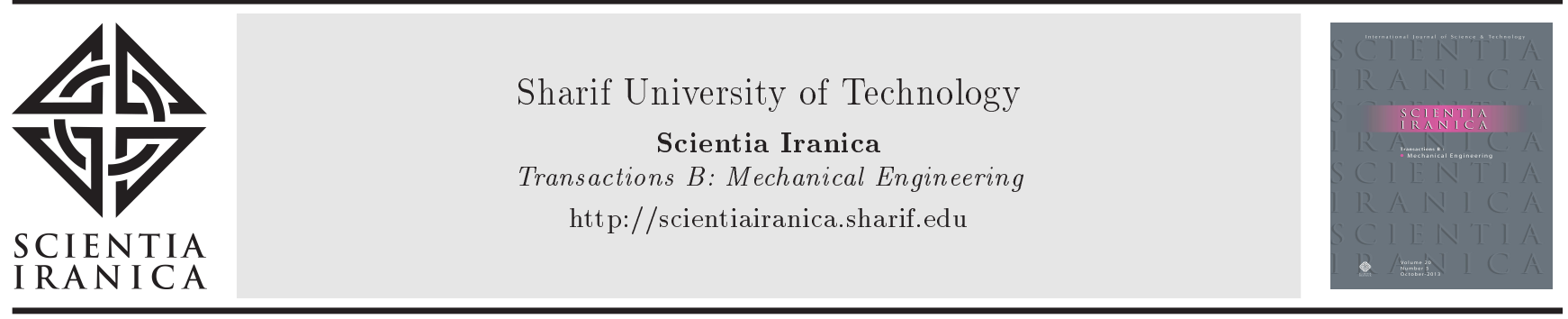

\title{
A modified indicial functions approximation for nonlinear aeroelastic analysis
}

\author{
M. Nejati ${ }^{\mathrm{a}}$, S. Shokrollahi ${ }^{\mathrm{a}, *}$, Sh. Shams ${ }^{\mathrm{b}}$, and R. Torkaman ${ }^{\mathrm{a}}$ \\ a. Department of Aerospace Engineering, Malek-Ashtar University of Technology, Tehran, Iran. \\ b. Department of Aerospace Engineering, Faculty of New Sciences and Technologies, University of Tehran, Tehran, Iran.
}

Received 6 January 2018; received in revised form 30 October 2018; accepted 26 January 2019

\section{KEYWORDS \\ Indicial aerodynamics; Subsonic flow; \\ Limit cycle oscillation; Nonlinear aeroelastic; \\ High aspect ratio.}

\begin{abstract}
The nonlinear dynamic response, Limit Cycle Oscillations (LCOs), of High-Aspect-Ratio (HAR) wings using novel indicial aerodynamics in subsonic flow was investigated. Using the nonlinear beam theory, the structural model was derived with in-plane and out-of-plane bending and torsional motions, all nonlinearities up to cubic order arising from large deformation, mass distribution, and cross-sectional mass imbalance. Based on new approximations of the indicial functions, a comprehensive unsteady aerodynamic model was used. Coupling such indicial aerodynamics to nonlinear structural equations can result in a unified nonlinear aeroelastic formulation in both incompressible and subsonic compressible flows. The effects of flight conditions, wing tip initial disturbances, Stiffness Ratio (SR) between bending modes, and nonlinearity due to inertia and cross-sectional mass imbalance on the characteristics of LCO are discussed. The results showed that compressibility could affect the LCO boundary up to 12 percent, which implied that appropriate Mach-dependent aerodynamics was required to achieve a more reasonable and realistic description of dynamic behavior of the system. It was observed that the presence of LCO before the linear flutter speed depended on the initial disturbances as well as wing characteristics.
\end{abstract}

(C) 2020 Sharif University of Technology. All rights reserved.

\section{Introduction}

High-Aspect-Ratio (HAR) and highly-flexibility lifting surfaces can be taken into account as the main features of High-Altitude Long-Endurance (HALE) aircraft. Undergoing large deformations due to aerodynamic loads, such wings exhibit nonlinear dynamic behaviors, which cannot be described by classical aeroelastic methods. Therefore, the nonlinearities caused by large deformations should be considered to study the

*. Corresponding author.

E-mail addresses: M_nejati@mut.ac.ir (M. Nejati);

s_shokrollahi@mut.ac.ir (S. Shokrollahi);

Shahrokh.shams@ut.ac.ir (Sh.Shams);

en.torkaman.aerospace@gmail.com (R.Torkaman) nonlinear phenomena such as Limit Cycle Oscillations (LCOs), bifurcation, chaotic vibration, and internal resonance of such wings. LCO is a steady-state periodic oscillation which may accrue at velocities both above and below the linear flutter speed, depending upon the dynamic of system, nonlinear mechanism, and the magnitude of applied disturbance. During the last decades, various researches have been carried out on the nonlinear aeroelastic problems, including twodimensional airfoils, single wings, and full aircraft. In order to investigate the aeroelastic behavior of HAR wings, continuous highly flexible beam models undergoing large deformations with the modified strip theory have widely been applied by researchers [1] Large deformations due to in-plane, out-of-plane, and torsional motions will introduce significant structural nonlinearity into the aeroelastic equations of motion. 
In fact, the nonlinear strain-displacement relations (geometric nonlinearity) are responsible for the appearance of nonlinear coupling terms in the bendingbending-torsion equations [2]. As a result of increasing aspect ratio, the importance of in-plane motion in the dynamic responses due to nonlinear coupling should also be included [3,4]. According to the above discussion, although the stability characteristics of HAR wings, e.g., flutter speed, may be determined by linear methods, nonlinear analysis is inevitable to investigate the nonlinear phenomena and study the dynamic responses.

Appropriate structural and aerodynamic modeling may result in an accurate aeroelastic analysis. The nonlinear beam theory is generally adopted to describe large deformations and the related geometrical nonlinearity effects of HAR wings. Geometric nonlinearities may generally be classified into the following types: large displacement-large rotation-small strain, large displacement-small rotation-small strain, and large displacement-large rotation-large strain, among which the first one is commonly considered in the HAR wings [1]. Nonlinear moving beam and geometrically exact intrinsic beam are two types of basic structural models. Hodges and Dowell [5] presented the nonlinear moving beam model with quadratic nonlinearities for twisted non-uniform rotor blades. However, the model did not have enough accuracy for large deflections, because the resulting coupled equations of motion included only second-order nonlinear terms [6]. Considering some third-order nonlinear terms, Rosen and Friedmann [7] obtained more accurate equations than those of Hodges and Dowell. Crespo da Silva and Glynn [8] developed a set of cubic nonlinear differential equations including the coupled bending-bendingtorsional motions of Euler-Bernoulli beams with arbitrary boundary conditions. The derived equations included the nonlinear effects due to curvature and inertia and they were suitable for nonlinear analysis of moderately large oscillations. Comprehensive nonlinear equations describing the extensional-bendingbending-torsional motions of three-dimensional beams were derived by Pai and Nayfeh [9]. The findings indicated that although the geometric nonlinearities dominated the inertia nonlinearities for the lowfrequency modes, the inertia nonlinearities played a more significant role in high-frequency modes [10]. The primary form of geometrically exact intrinsic beam model was first developed by Hodges [11]. The model provided a geometrically exact, fully intrinsic dynamic formulation for moving beam.

Various aerodynamic theories such as indicial response theory, Euler or Navier-Stokes CFD techniques, and unsteady vortex-lattice method can be used to calculate aerodynamic loads [12-17]. Classical aerodynamic loads such as Wagner, Theodorsen, and Green- berg models have limited practical applications due to the effects of compressibility and restriction of the solution method to the frequency domain. On the other hand, numerical methods based on computational fluid dynamics may also be impractical and inefficient in common aeroelastic problems due to solution complexity and requirement of extremely large amounts of computational resources. The indicial function method has attracted more attention to determining unsteady aerodynamic loads undergoing arbitrary motions in the compressible flow field because of its practical and convenient computational forms leading to suitable formulations in aeroelasticity problems. Mazelsky [18,19] and Mazelsky and Drischler [20] approximated the indicial response functions with a series of four exponential terms at specified Mach numbers (i.e. 0.5, 0.6, and 0.7). Leishman [15] obtained the unsteady indicial aerodynamic functions approximated by a series of three exponential terms, due to step changes in the angle of attack and pitching rate in subsonic compressible flow. Marzocca et al. [21,22] determined the unsteady aerodynamic loads for an oscillating airfoil using linear and nonlinear indicial functions in the incompressible and compressible subsonic, transonic, supersonic, and hypersonic flight speed regimes. Farsadi and Javanshir [23] developed a Mach-dependent exponential approximation of indicial functions in the range of $0.5 \leq M \leq 0.8$ for subsonic compressible flow. Based on alternative approximations of indicial functions, Nejati et al. [24,25] developed a comprehensive, efficient, and Mach-dependent unsteady indicial aerodynamic model, which was valid throughout the entire subsonic flow, i.e. $0 \leq M \leq 0.8$. Despite the fact that the unsteady aerodynamic loads based on the indicial response method are often determined for 2-D airfoils, they can be generalized to $3-\mathrm{D}$ problems. To this end, the modified strip theory along with the modified lift curve slope is used to include $3-\mathrm{D}$ effects for the finitespan wings [26,27].

Extensive studies have been carried out on nonlinear aeroelasticity and the corresponding phenomena such as LCO, internal resonance, bifurcation, and chaotic motion of flexible HAR wings. Tang and Dowell [28] investigated the aeroelastic response and presence of LCO of an HAR wing both theoretically and experimentally. They adopted the ONERA stall model and Hodges-Dowell equations to describe the structural and aerodynamic nonlinearities, respectively. Patil et al. [3] used geometrically exact structural analysis coupled to finite-state unsteady aerodynamics with stall to numerically analyze LCOs in HAR wings. They found that a critical disturbance value should be suggested at a given speed and a critical speed reached at a given disturbance magnitude to initiate LCO. Strganac et al. [4] studied the nonlinear behaviors and bifurcation characteristics of highly flex- 
ible wings undergoing moderate-to-high deformations. They used Crespo da Silva beam theory and NavierStokes method to develop the governing nonlinear aeroelastic equations. Shams et al. studied the flexuraltorsional as well as flexural-flexural-torsional nonlinear aeroelastic response of slender wings using the Crespo da Silva nonlinear beam model and unsteady linear aerodynamic strip theory based on the Wagner function $[29,30]$. Jian and Jinwu [31] used the geometrically exact, fully intrinsic beam model [32] attached to a nonlinear dynamic stall model including drag to derive a first-order, state-space model for nonlinear aeroelastic analysis of HALE wings. Eskandari et al. [33] investigated the effect of different parameters including Stiffness Ratio (SR) and mass ratio as well as mass imbalance on divergence and flutter speed along with LCO of HAR wings. They employed the HodgesDowell moving beam and quasi-steady aerodynamics to derive the governing aeroelastic equations. Badiei et al. [34] investigated the aeroelastic responses of HAR wings in the stall and post-stall regions of incompressible flow. They derived a nonlinear aeroelastic model using the nonlinear and nonplanar motions of EulerBernoulli beam theory and an unsteady aerodynamic static stall model. Dardel et al. [35] investigated the effect of angle of attack on aeroelastic features of HAR wings with structural nonlinearities, including HodgesDowell moving beam in unsteady subsonic compressible flow, based on Wagner function. Xiao et al. [36] studied the limit cycle oscillation of an isotropic HAR wing through an unsteady incompressible aerodynamic model. They showed that the use of the first two modes of out-of-plane and torsional motions was good enough to determine the instability. Koohi et al. $[37,38]$ developed a modified aeroelastic model to calculate the stability of composite HAR wings using a beam theory presented by Yuan and Friedmann [39] and an unsteady aerodynamic model based on Jones's approximation and ONERA dynamic stall. They concluded that higher-order terms in structural equations undergoing large deflection should be taken into account. Jung et al. [40] developed a coupled CFD-CSD method to predict aeroelastic static deflections and dynamic aeroelastic behaviors of HAR wings. Bakhtiari-Nejad et al. [41] studied linear and nonlinear aeroelastic behaviors of HAR wings using a third-order nonlinear beam model coupled to Wagner state-space model. They found that geometric structural nonlinearity had a detrimental effect on the flutter behavior. Using a method of joining three-dimensional and onedimensional finite elements, a reduced-order beam model was developed by Hosseini and Hodges [42] to investigate the nonlinear aeroelasticity of HAR wings. Computational cost efficiency and high accuracy were two advantages of their model. They also adopted finite-state induced flow theory of Peters to model the unsteady aerodynamic loads. Farsadi et al. [43] geometrically studied the nonlinear aeroelastic behavior of pre-twisted HAR wings. The structure was modeled as thin walled beams and the approximation of the Wagner's function in time domain was used to describe unsteady aerodynamic loads in the incompressible flow regime. Proposing an alternative simulation approach to the nonlinear ONERA aerodynamic model, leading to a reduction in the computational cost, $\mathrm{Xu}$ et al. [44] studied the nonlinear aeroelastic behaviors of a slender wing without store and the wing-pylon-store system. Nejati et al. [25] developed a nonlinear aeroelastic model to investigate nonlinear aeroelastic behavior of HAR wings. They adopted nonlinear 3-D EulerBernoulli theory and unsteady indicial aerodynamics to model the out-of-plane bending and torsional motions of a highly flexible wing in the subsonic compressible flight speed regime. As mentioned earlier, the in-plane bending mode is more significant than other modes in the dynamic behavior of HAR wings. Therefore, it should be taken into account in the governing aeroelastic equations of motion. Following the previous work, the nonlinear aeroelastic behavior of HAR wings, including the in-plane and out-of-plane bending and torsional motions, in subsonic flow is investigated in the present study.

The main objective of the present research is development of a unique formulation to investigate the nonlinear behavior of HAR wings over the entire range of subsonic flow, including incompressible and compressible cases. Following the nonlinear moving beam approach applied by Nayfeh and Pai [2], a nonlinear structural model is derived, focused on the effect of cross-sectional mass imbalance, including the in-plane and out-of-plane bending as well as torsional motions. The concept of indicial function is adopted to determine the unsteady aerodynamic loads [24]. A unique representation of the indicial functions for any axis of rotation, unified aerodynamic formulations for both incompressible and subsonic compressible flow, and a new and efficient form of unsteady aerodynamic loads appropriate to state-space analysis are some advantages of the applied method. To derive the governing aeroelastic equations of motion, the indicial aerodynamic loads are introduced into the governing structural equations. The resulting partial differential equations contain the linear and nonlinear bendingtorsion and nonlinear bending-bending coupling terms, the linear and nonlinear contributions due to mass imbalance in the cross section, and both quadratic and cubic nonlinearities due to curvature and inertia, and they are valid over the entire range of subsonic flow. To verify the nonlinear aeroelastic equations and to investigate the aeroelastic behavior of HAR wings, including flutter instability and response analysis, the linear eigenvalue and the time-domain linear/nonlinear 
response analysis are applied to two wing models, respectively, for both incompressible and subsonic compressible flows.

The flutter boundary, flutter and post-flutter time responses, LCO, and phase plane diagrams are studied for various Initial Tip Disturbances (ITDs) and different flight conditions. The effects of compressibility, flight conditions including altitude and air speed, various ITDs, the SR between out-of-plane and in-plane bending modes, nonlinearity due to inertia, and cross-sectional mass imbalance on the nonlinear dynamic response are also discussed in this paper.

\section{Equations of motion}

Development and suitable combination of proper aerodynamic and structural models are the main steps towards achieving nonlinear equations of motion. In the following subdivision, it is discussed how to derive the first-order nonlinear differential aeroelastic equations in the state-space form, valid for large oscillations of metallic HAR wings over the entire range of subsonic flow.

\subsection{Structural model}

Here, a brief explanation is provided as to how the

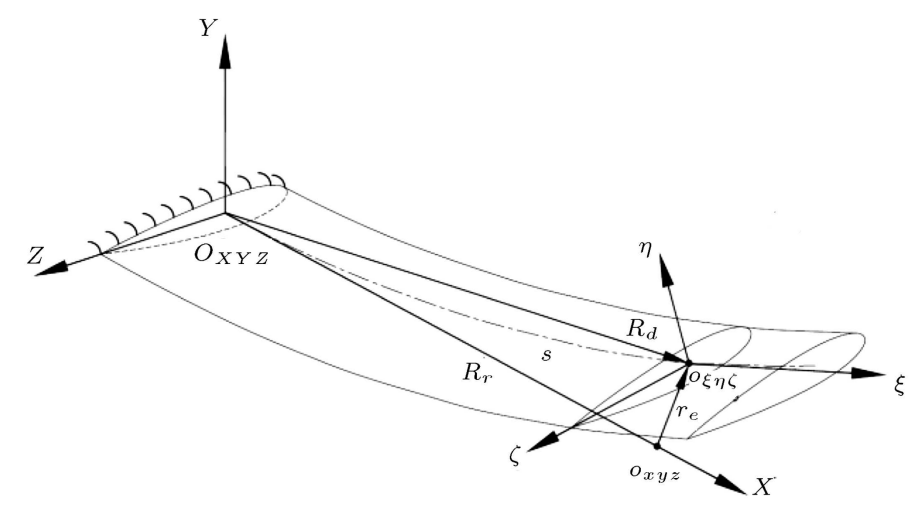

(a)

Figure 1. Undeformed and deformed coordinate systems: (a) Wing configuration and (b) two successive Euler angles.

nonlinear structural model is developed. The reader is referred to [2] for further details. Consider an initially straight wing of length $L$ and mass per unit length $m$, as shown in Figure 1(a). Two coordinate systems can be employed in order to determine the whole kinematics of the system: the Cartesian coordinate system $x y z$ describing the undeformed geometry and the orthogonal curvilinear coordinate system $\xi \eta \zeta$ depicting the deformed geometry. Let $u, v$, and $w$ denote the displacements of an arbitrary point located on the cross section along $x, y$, and $z$ directions, respectively, and $s$ denote the undeformed length from the root of the wing. According to Figure 1(b), the coordinate systems can be transformed from the undeformed position to the deformed one under two successive rotations of $\gamma$ and $\phi$ about the $n$ and $\zeta$ axes, respectively. The unit vectors of these two coordinate systems are then related as:

$$
\left\{\begin{array}{lll}
\mathbf{i}_{\xi} & \mathbf{i}_{\eta} & \mathbf{i}_{\zeta}
\end{array}\right\}^{T}=[\mathbf{T}]\left\{\begin{array}{lll}
\mathbf{i}_{x} & \mathbf{i}_{y} & \mathbf{i}_{z}
\end{array}\right\}^{T},
$$

where the transformation matrix $[\mathbf{T}]$, which is a function of four dependent variables $u, v, w$, and $\phi$ can be obtained using the relation between the unit vectors of coordinate systems along with geometric relations between the undeformed and deformed differential elements, as shown in Figure 2.

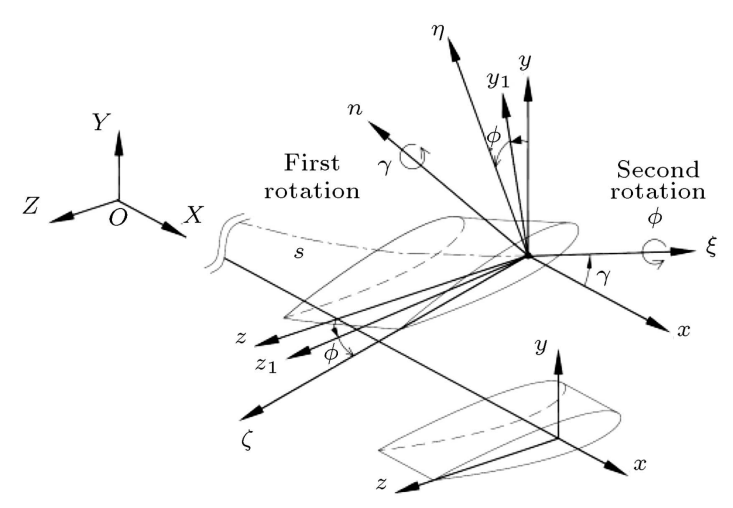

(b)

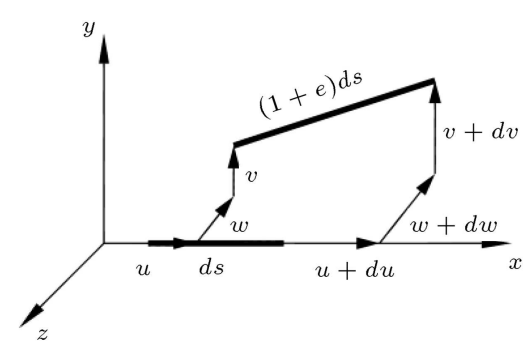

(a)

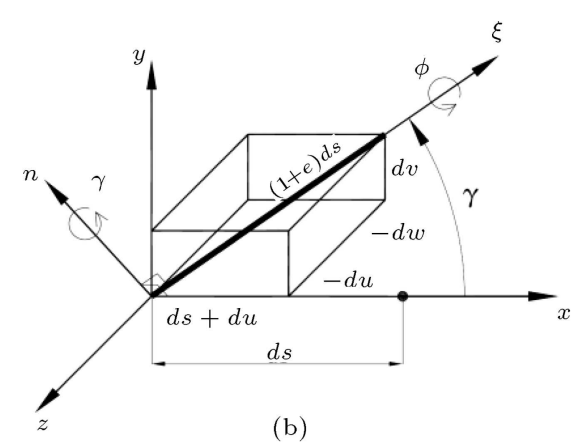

(b)

Figure 2. Geometrical relations between the undeformed and deformed differential elements: (a) Displacements and (b) Euler angles. 
In the absence of warping, a differential element of the wing can be supposed as a thin rigid plate with infinitesimal thickness $d s$ and a constant finite area. Hence, the motion of such an element may be expressed by three translational displacements $u, v$, and $w$ and two rotations $\gamma$ and $\phi$. According to Figure 2(a), the relation between the axial strain $e$ and the displacement filed is:

$$
e=\sqrt{\left(1+u^{\prime}\right)^{2}+{v^{\prime}}^{2}+{w^{\prime}}^{2}}-1
$$

where the prime indicates the derivative with respect to $s$. Under the assumption of inextensionality and neglecting some higher-order terms, it can be shown that $u$ is a second-order quantity as:

$$
u=-\frac{1}{2} \int_{0}^{s}\left(v^{\prime 2}+w^{\prime 2}\right) d s .
$$

The component $T_{1 j}, j=1,2,3$, can be obtained by projecting the unit deformed vector shown in Figure 2(b) along the unit vectors of the Cartesian coordinate system $x y z$.

$$
T_{11}=\frac{1+u^{\prime}}{1+e}, \quad T_{12}=\frac{v^{\prime}}{1+e}, \quad T_{13}=\frac{w^{\prime}}{1+e} .
$$

Following the transformation approach using two Euler angles and known components $T_{1 j}, j=1,2,3$, the transformation matrix is defined by Eq. (5) as shown in Box I, where the trigonometric functions can be written as:

$$
\cos \phi=\frac{\sqrt{\left(1+u^{\prime}\right)^{2}+{v^{\prime}}^{2}}}{1+e}, \quad \sin \phi=\frac{-w^{\prime}}{1+e} .
$$

Moreover, the deformed curvatures and the angular velocity of the deformed system can be derived by differentiating Eq. (1) with respect to $s$, considering the relation between the curvatures and the unit vectors of the orthogonal curvilinear system, and using the Kirchhoff's kinetic analogy [2].

$$
\begin{aligned}
& \rho_{\xi}=\sum_{i=1}^{3} T_{2 i}^{\prime} T_{3 i}, \quad \rho_{\eta}=-\sum_{i=1}^{3} T_{1 i}^{\prime} T_{3 i}, \\
& \rho_{\zeta}=\sum_{i=1}^{3} T_{1 i}^{\prime} T_{2 i},
\end{aligned}
$$

$$
\begin{aligned}
& \omega_{\xi}=\sum_{i=1}^{3} \dot{T}_{2 i} T_{3 i}, \quad \omega_{\eta}=-\sum_{i=1}^{3} \dot{T}_{1 i} T_{3 i}, \\
& \omega_{\zeta}=\sum_{i=1}^{3} \dot{T}_{1 i} T_{2 i},
\end{aligned}
$$

where $\rho_{i}$ and $\omega_{i}, i=\zeta, \eta, \zeta$ indicate curvature and angular velocity, respectively, along the orthogonal curvilinear coordinate system $\zeta \eta \zeta$ and the overdot denotes the derivative with respect to time. After determining the whole kinematic relations of the system, the energy expressions can now be obtained. If the deformed position vector of an arbitrary point on the cross section is expressed as:

$$
\mathbf{R}=(s+u) i_{x}+v i_{y}+w i_{z}+y i_{\eta}+z i_{\zeta},
$$

then, variation of the kinetic energy is defined by:

$$
\delta T=-\int_{0}^{L} \int_{A} \rho \ddot{\mathbf{R}} . \delta \mathbf{R} d A d s
$$

where $\rho$ and A denote the material density and the area of the cross section, respectively. Note that the concept of orthogonal virtual relations is used in deriving the above variation. Nayfeh and Pai [2] assumed that the reference point (e.g., the shear center) coincided with the mass centroid. Hence, the first moment of inertia matrix became zero, i.e. $\left[\mathbf{J}_{1}\right]=0$, in their research. However, this matrix, which contains the socalled mass imbalance components, is not always equal to zero. Mass imbalance is often caused by differential placement of the center of mass and elastic axis and it plays a significant role in aeroelastic phenomena of aircraft wings. It can introduce both linear and nonlinear coupling terms into governing equations of motion. Considering the whole mass imbalance effects in deriving the nonlinear structural model may be the main difference between the present study and other investigations. As a result of the symmetry of cross section about $\zeta$ axis and the presence of mass imbalance in the chord-wise direction, the first moment of inertia matrix is given by:

$$
\left[\mathbf{J}_{1}\right]=m e_{z}\left[\begin{array}{ccc}
0 & 1 & 0 \\
-1 & 0 & 0 \\
0 & 0 & 0
\end{array}\right]
$$

$$
[\mathbf{T}]=\left[\begin{array}{ccc}
1 & 0 & 0 \\
0 & \cos \phi & \sin \phi \\
0 & -\sin \phi & \cos \phi
\end{array}\right]\left[\begin{array}{ccc}
T_{11} & T_{12} & T_{13} \\
-T_{12} & T_{11}+T_{13}^{2} /\left(1+T_{11}\right) & -T_{12} T_{13} /\left(1+T_{11}\right) \\
-T_{13} & -T_{12} T_{13} /\left(1+T_{11}\right) & T_{11}+T_{12}^{2} /\left(1+T_{11}\right)
\end{array}\right]
$$


where $e_{z}$ denotes the distance between the center of mass and the elastic center. Local strain and stress values are used in order to fully account for geometric nonlinearity. According to these values, variation of the elastic energy is given by:

$$
\begin{aligned}
\delta \Pi= & \int_{0}^{L} \int_{A}\left(\sigma_{\xi \xi} \delta \epsilon_{\xi \xi}+\sigma_{\xi \eta} \delta \epsilon_{\xi \eta}+\sigma_{\xi \zeta} \delta \epsilon_{\xi \zeta}+\sigma_{\eta \zeta} \delta \epsilon_{\eta \zeta}\right. \\
& \left.+\sigma_{\eta \eta} \delta \epsilon_{\eta \eta}+\sigma_{\zeta \zeta} \delta \epsilon_{\zeta \zeta}\right) d A d s
\end{aligned}
$$

where $\sigma_{i j}$ denotes local stress and local strain components, i.e. $\epsilon_{i j}$, can be determined by the relationship between the local displacement field and its derivative with respect to $s$. The local strain is therefore expressed as:

$$
\begin{aligned}
& \epsilon_{\xi \xi}=e-y \rho_{\zeta}+z \rho_{\eta}, \quad \epsilon_{\xi \eta}=-z \rho_{\xi}, \\
& \epsilon_{\xi \zeta}=y \rho_{\xi} \\
& \epsilon_{\eta \eta}=\epsilon_{\eta \zeta}=\epsilon_{\zeta \zeta}=0 .
\end{aligned}
$$

As mentioned earlier, there are four dependent variables, namely $u, v, w$, and $\phi$. Assuming linear viscous damping, the non-conservative expressions can be written as:

$$
\begin{aligned}
\delta W_{n c}= & \int_{0}^{L}\left(-c_{u} \dot{u} \delta u-c_{v} \dot{v} \delta v-c_{w} \dot{w} \delta w-c_{\phi} \dot{\phi} \delta \theta_{1}\right. \\
& \left.+Q_{u} \delta u+Q_{v} \delta v+Q_{w} \delta w+Q_{\phi} \delta \theta_{1}\right)
\end{aligned}
$$

where $c_{i}$ and $Q_{i}(i=u, v, w$, and $\phi)$ are structural damping ratios and distributed external loads per unit of length, respectively, and $\delta \theta_{1}$ denotes the virtual rigid-body rotation with respect to the $\xi$ axis.

It should be reminded that under the inextensionality assumption, the dependent variables are reduced to three, namely $v, w$, and $\phi$. Hence, the equation governing $u$ must be treated as a constraint. Moreover, the variable $\phi$ does not represent the real twist angle. The real twisting angle, which can be determined using the twisting curvature and kinematic boundary conditions, is as follows [9]:

$$
\phi=\alpha+\frac{1}{2} v^{\prime} w^{\prime}-\int_{0}^{s} v^{\prime \prime} w^{\prime} d s .
$$

The transcendental governing equations of motion for bending-bending-torsional vibrations of a cantilevered wing with associated boundary conditions can now be derived using the extended Hamilton's principle.

$$
\int_{t_{1}}^{t_{2}}\left(\delta T-\delta \Pi+\delta W_{n c}\right) d t=0 .
$$

In order to derive the polynomial form of the governing equations from the transcendental ones for small but finite oscillations about the undeformed position, one can assume that the order of dependent variables and their derivatives is $O(\varepsilon)$, where $\varepsilon$ is a small dimensionless parameter $(\varepsilon \ll 1)$. It can be shown that the axial deformation $u$ is of course $O\left(\varepsilon^{2}\right)$ for the inextensional case. All transcendental terms in governing equations are then expanded in a Taylor series and they keep nonlinear terms up to $\left(\varepsilon^{3}\right)$. After the laborious and complex mathematical operations along with some algebraic manipulations, the thirdorder nonlinear equations of motion describing in-plane bending $(w)$, out-of-plane bending $(v)$, and torsional motion $(\alpha)$ are obtained as follows:

$$
\begin{aligned}
& m \ddot{w}+c_{w} \dot{w}+D_{\eta} w^{i v}=G_{w}+Q_{w}, \\
& m \ddot{v}-m e_{z} \ddot{\alpha}+c_{v} \dot{v}+D_{\zeta} v^{i v}=G_{v}+Q_{v}, \\
& j_{\xi} \ddot{\alpha}-m e_{z} \ddot{v}+c_{\alpha} \dot{\alpha}-D_{\xi} \alpha^{\prime \prime}=G_{\alpha}+Q_{\alpha},
\end{aligned}
$$

where:

$$
\begin{aligned}
G_{w}= & D_{\xi}\left(v^{\prime \prime} \alpha^{\prime}\right)^{\prime}+\left(D_{\eta}-D_{\zeta}\right) \\
& {\left[\left(v^{\prime \prime} \alpha+w^{\prime \prime} \alpha^{2}\right)^{\prime}+v^{\prime \prime \prime} \int_{0}^{s} v^{\prime} w^{\prime \prime} d s\right]^{\prime} } \\
& -D_{\eta}\left[\left(w^{\prime} w^{\prime \prime}+v^{\prime} v^{\prime \prime}\right)^{\prime} w^{\prime}\right]^{\prime} \\
& +j_{\eta}\left[\left(v^{\prime} \dot{v}^{\prime}+w^{\prime} \dot{w}^{\prime}\right)^{\bullet} w^{\prime}\right]^{\prime} \\
& -\frac{1}{2}\left\{w^{\prime} \int_{L}^{s}\left[m \int_{0}^{s}\left(v^{\prime 2}+w^{\prime 2}\right)^{\bullet \bullet} d s\right] d s\right\}^{\prime} \\
& -j_{\xi}\left[\left(\dot{\alpha}^{\prime}+\dot{v} w^{\prime}-\int_{0}^{s}\left(v^{\prime \prime} w^{\prime}\right)^{\bullet} d s\right)^{\prime}\right]^{\prime} \\
& -m e_{z}\left(v^{\prime} \ddot{v}+w^{\prime} \ddot{w}\right)^{\prime} \\
& -\left(j_{\zeta}-j_{\eta}\right)\left[\left(\dot{v}^{\prime} \alpha+\dot{w}^{\prime}\right]^{\prime}-m e_{z}^{2}+\dot{v}^{\prime} \int_{0}^{s} w^{\prime} w^{\prime \prime} d s\right)^{\bullet}
\end{aligned}
$$




$$
\begin{aligned}
& +\frac{1}{2} m e_{z}\left({v^{\prime}}^{2}+2{w^{\prime}}^{2}+\alpha^{2}-2 \alpha \int_{0}^{s} v^{\prime \prime} w^{\prime} d s\right)^{\bullet \bullet} \\
& +j_{\eta} \ddot{w}^{\prime \prime}, \\
& G_{v}=-D_{\zeta}\left[v^{\prime}\left(v^{\prime \prime} v^{\prime}+w^{\prime} w^{\prime \prime}\right)^{\prime}\right]^{\prime} \\
& -\left(D_{\eta}-D_{\zeta}\right)\left[\left(v^{\prime \prime} \alpha^{2}-w^{\prime \prime} \alpha\right)^{\prime}+w^{\prime \prime \prime} \int_{0}^{s} v^{\prime \prime} w^{\prime} d s\right]^{\prime} \\
& -D_{\xi}\left(w^{\prime \prime} \alpha^{\prime}\right)^{\prime}-\frac{1}{2} m\left\{v^{\prime} \int_{L}^{s} \int_{0}^{s}\left(v^{\prime 2}+w^{\prime 2}\right)^{\bullet \bullet} d s d s\right\}^{\prime} \\
& +j_{\xi}\left[\dot{w}^{\prime}\left(\dot{\alpha}+\dot{v}^{\prime} w^{\prime}-\int_{0}^{s}\left(v^{\prime \prime} w^{\prime}\right)^{\bullet} d s\right)\right]^{\prime}+\left(j_{\eta}-j_{\zeta}\right) \\
& {\left[\left(\dot{v}^{\prime} \alpha^{2}-\dot{w}^{\prime} \alpha+\dot{w}^{\prime} \int_{0}^{s} v^{\prime \prime} w^{\prime} d s\right)^{\bullet}-\dot{v}^{\prime} w^{\prime} \dot{w}^{\prime}\right]^{\prime}} \\
& +\left[j_{\zeta} v^{\prime}\left(v^{\prime} \dot{v}^{\prime}+w^{\prime} \dot{w}^{\prime}\right)^{\bullet}\right]^{\prime} \\
& -m e_{z}\left\{v^{\prime} \int_{L}^{s}\left(w^{\prime}-v^{\prime} \alpha\right)^{\bullet \bullet} d s\right\}^{\prime}
\end{aligned}
$$

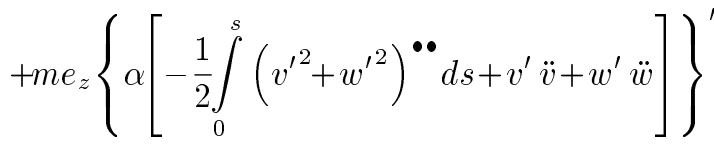

$$
\begin{aligned}
& -\frac{1}{2} m e_{z}\left(v^{\prime 2} \alpha+\frac{1}{3} \alpha^{3}-2 \int_{0}^{s} v^{\prime} w^{\prime \prime} d s\right)^{\bullet \bullet}+j_{\zeta} \ddot{v}^{\prime \prime}, \\
& G_{\alpha}=\left(D_{\zeta}-D_{\eta}\right)\left[\left(v^{\prime \prime 2}-w^{\prime \prime 2}\right) \alpha-v^{\prime \prime} w^{\prime \prime}\right] \\
& +\left(j_{\eta}-j_{\zeta}\right)\left[\left(\dot{v}^{\prime 2}-\dot{w}^{\prime 2}\right) \alpha-\dot{w}^{\prime} \dot{v}^{\prime}\right] \\
& +j_{\xi}\left[\int_{0}^{s}\left(v^{\prime \prime} w^{\prime}\right)^{\bullet \bullet} d s-\left(\dot{v}^{\prime} w^{\prime}\right)^{\bullet}\right] \\
& +m e_{z}\left(\alpha-\int_{0}^{s} v^{\prime \prime} w^{\prime} d s\right) \ddot{w} \\
& +m e_{z}\left[\frac{1}{2} v^{\prime} \int_{0}^{s}\left(v^{\prime 2}+w^{\prime 2}\right)^{\bullet \bullet} d s-\frac{1}{2}\left(v^{\prime 2}+\alpha^{2}\right) \ddot{v}\right]
\end{aligned}
$$

Considering the fact that rotary inertia is of order of two and neglecting the mass imbalance effects, the same equations as those derived by Crespo da Silva can be obtained [8]. Moreover, the same equations as developed by Strganac et al. [4] and Abbas et al. [45] can be derived by ignoring nonlinear mass imbalance terms and the in-plane motion, respectively.

\subsection{Indicial aerodynamic model}

The unsteady aerodynamic loads can be determined using the indicial function concept on a wing undergoing any arbitrary motions via Fourier superposition and Duhamel integral [26]. While in the incompressible flight speed regime, a single indicial function, socalled Jones approximation of Wagner's function, is used to calculate the unsteady loads, four Machdependent indicial functions are required to evaluate the unsteady lift and moment in the compressible flight speed regime. This results in different formulations and consequently, different aerodynamic models in the incompressible and subsonic compressible flows. On the other hand, the indicial functions often have various Mach-dependent exponential terms, which cause some complexity in computing them in different positions as they are approximated initially. To obtain a unique representation of the indicial functions, which supply a more convenient form while transferring to an arbitrary axis of rotation throughout the entire range of subsonic flight speed regime, the novel, efficient, and Machdependent approximations of the indicial functions are developed using the analytical and available numerical data. The detailed description of process of approximating the indicial functions has been reported in a previous work by the authors [24,25]. Hence, in the following, only the indicial aerodynamic loads are represented for applying to the governing structural equations. For two-dimensional airfoils, as shown in Figure 3, the indicial functions are generally expressed in exponential form as:

$$
\phi_{k}(t, M)=b_{0}(M)+\sum_{j=1}^{3} b_{j k}(M) e^{-\beta_{j} t},
$$

where $M$ is the Mach number; $\phi_{c \alpha}(\tau), \phi_{c m}(\tau), \phi_{c q}(\tau)$, and $\phi_{c m q}(\tau)$ represent indicial lift and moment func-

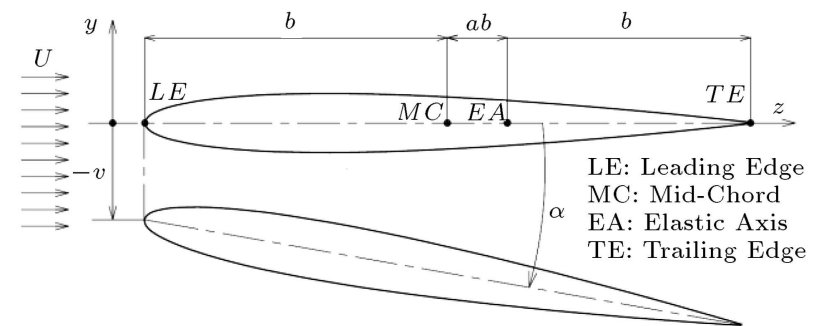

Figure 3. Vertical translation and pitching about an axis through the leading edge. 
Table 1. Exponential coefficients for approximating the indicial lift and moment.

\begin{tabular}{ccccc}
\hline Indicial function & $\boldsymbol{\beta}_{\mathbf{0}}$ & $\boldsymbol{\beta}_{\mathbf{0 1}}$ & $\boldsymbol{\beta}_{\mathbf{2}}$ & $\boldsymbol{\beta}_{\mathbf{3}}$ \\
\hline$\phi_{c \alpha}$ & 0 & 0.0754 & 0.3720 & 1.890 \\
\hline
\end{tabular}

tions about the elastic axis due to a sudden change in the vertical translation velocity and the pitching rate at the leading edge, respectively; $b_{0}$ and $b_{j k}$ are the indicial coefficients, which are approximated throughout the entire range of subsonic compressible flow; and $\beta_{j}$ represents Mach-independent exponential coefficients of the indicial lift function due to a change in the angle of attack at Mach number of 0.5, as shown in Table 1.

As the indicial functions are usually approximated about the axis of rotation passing through the leading edge, one can determine them about an arbitrary axis, e.g. elastic axis, only by modifying their constituent coefficients. If the axis of rotation is placed at a distance $(1+a) b$ from the leading edge, as shown in Figure 3, then we have:

$$
\begin{aligned}
& b_{j c \alpha}=\bar{b}_{j c \alpha}, \\
& b_{j c m}=\bar{b}_{j c m}+\frac{1}{2}(a+1) \bar{b}_{j c \alpha},
\end{aligned}
$$

$$
\begin{gathered}
b_{j c q}=\bar{b}_{j c q}-\frac{1}{2}(a+1) \bar{b}_{j c \alpha}, \\
b_{j c m q}=\bar{b}_{j c m q}+\frac{1}{2}(a+1)\left(\bar{b}_{j c q}-\bar{b}_{j c m}\right) \\
-\frac{1}{4}(a+1)^{2} \bar{b}_{j c \alpha},
\end{gathered}
$$

where $\bar{b}_{j}$ and $b_{j}$ are the constituent coefficients of the indicial functions about the leading edge and an axis which is located at $a b$ behind the midchord, respectively. The coefficient $\bar{b}_{j k}(j=1 \ldots 3$ and $k$ denotes the type of indicial function) is plotted continuously versus Mach number in Figure 4 [24].

It should be noted that compressibility has negligible effect on the behavior of indicial functions for Mach numbers less than 0.2 [15,24,25]; therefore, the indicial functions in the incompressible case can be used to obtain the unsteady aerodynamic loads in this flight speed range.

In order to have the same representation of aerodynamic loads over the entire subsonic flow regime, one can substitute the single Wagner's function with four indicial ones, including the same known exponential coefficients as mentioned above. The coefficients approximating the indicial lift and moment in the

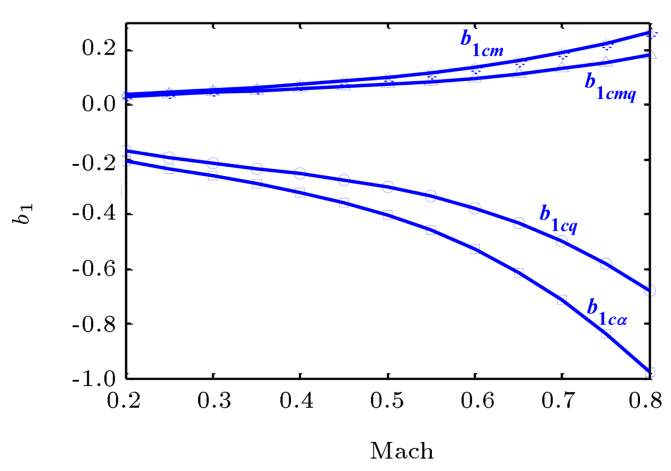

(b) Coefficients of $\bar{b}_{1}$

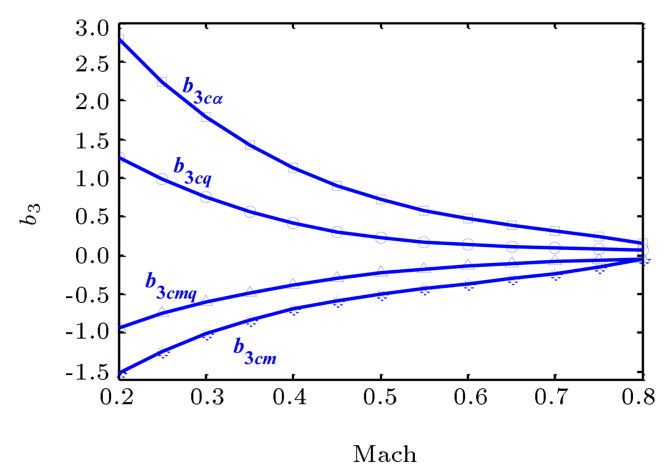

(d) Coefficients of $\bar{b}_{3}$

Figure 4. Coefficients of the indicial functions with plunging and pitching about the leading edge at $0.2 \leq M \leq 0.8$. 
incompressible flow are also determined based on the same procedure mentioned above and shown in Table 2.

An appropriate and comprehensive representation of unsteady aerodynamic loads using such indicial functions can be express as:

$$
\begin{aligned}
& L(t)=\frac{C_{L_{\alpha}} q S}{U}\left\{\dot{v}(t) \phi_{c \alpha}(0)+v(t) \dot{\phi}_{c \alpha}(0)\right. \\
& -v(0) \dot{\phi}_{c \alpha}(t)+2 b \dot{\alpha}(t) \phi_{c q}(0) \\
& +\left[U \phi_{c \alpha}(0)+2 b \dot{\phi}_{c q}(0)\right] \alpha(t) \\
& -2 b \alpha(0) \dot{\phi}_{c q}(t)+\sum_{j=1}^{3} \lambda_{v 1 j} B_{1 j} \\
& \left.+\sum_{j=1}^{3} \lambda_{\alpha 1 j} B_{2 j}\right\} \\
& M_{e a}(t)=\frac{C_{L_{\alpha}} q S c}{U}\left\{\dot{v}(t) \phi_{c m}(0)+v(t) \dot{\phi}_{c m}(0)\right. \\
& -v(0) \dot{\phi}_{c m}(t)+2 b \dot{\alpha}(t) \phi_{c m q}(0) \\
& +\left[U \phi_{c m}(0)+2 b \dot{\phi}_{c m q}(0)\right] \alpha(t) \\
& -2 b \alpha(0) \dot{\phi}_{c m q}(t)+\sum_{j=1}^{3} \lambda_{v 2 j} B_{1 j} \\
& \left.+\sum_{j=1}^{3} \lambda_{\alpha 2 j} B_{2 j}\right\}
\end{aligned}
$$

where $C_{L_{\alpha}}$ denotes lift curve slope of $2 \mathrm{D}$ airfoil, $b$ is half chord, $S=2 b$ denotes the wing planform area per unit length, and $q$ and $U$ are the dynamic pressure and the free stream air speed, respectively:

$$
\begin{aligned}
& \lambda_{v 1 j}=b_{j c \alpha}\left(\beta_{j} \frac{U}{b}\right)^{2}, \\
& \lambda_{\alpha 1 j}=b \beta_{j}\left(\frac{U}{b}\right)^{2}\left(2 b_{j c q} \beta_{j}-b_{j c \alpha}\right),
\end{aligned}
$$

Table 2. Coefficients for approximating the indicial lift and moment at the Mach number of zero.

\begin{tabular}{ccccc}
\hline Indicial function & $\overline{\boldsymbol{b}}_{\mathbf{0}}$ & $\overline{\boldsymbol{b}}_{\mathbf{1}}$ & $\overline{\boldsymbol{b}}_{\mathbf{2}}$ & $\overline{\boldsymbol{b}}_{\mathbf{3}}$ \\
\hline $\bar{\phi}_{I c \alpha}$ & 1 & -0.2679 & -0.2274 & -0.0247 \\
$\bar{\phi}_{I c m}$ & -0.25 & 0.067 & 0.0568 & 0.0062 \\
$\bar{\phi}_{I c q}$ & 0.75 & -0.201 & -0.1706 & -0.0185 \\
$\bar{\phi}_{I c m q}$ & -0.25 & 0.0502 & 0.0426 & 0.0046 \\
\hline
\end{tabular}

$$
\begin{aligned}
& \lambda_{v 2 j}=b_{j c m}\left(\beta_{j} \frac{U}{b}\right)^{2}, \\
& \lambda_{\alpha 2 j}=b \beta_{j}\left(\frac{U}{b}\right)^{2}\left(2 b_{j c m q} \beta_{j}-b_{j c m}\right),
\end{aligned}
$$

and auxiliary state-apace variables, $B$, should satisfy the following first-order differential equations:

$$
\begin{aligned}
& \dot{B}_{i j}(t)+\left(\beta_{j} \frac{U}{b}\right) B_{i j}(t)=x_{i}(t), \\
& i=1,2, \quad j=1,2,3, \text { and } \\
& {\left[x_{1}, x_{2}\right]=[v, \alpha] .}
\end{aligned}
$$

Note that in the compressible unsteady aerodynamic theory, any concept of virtual mass is meaningless $[22,26,46]$. Since the unsteady aerodynamic loads, i.e. Eqs. (28) and (29), include only the circulatory portions, one should append the non-circulatory contributions while determining the unsteady incompressible lift and moment as follows $[22,26]$ :

$$
\begin{aligned}
L_{a}^{n c}(\tau)= & \frac{C_{L_{\alpha}} q b^{2}}{U^{2}}[\ddot{v}(\tau)+U \dot{\alpha}(\tau)-a b \ddot{\alpha}(\tau)], \\
M_{e a}^{n c}(\tau)= & \frac{C_{L_{\alpha}} q b^{3}}{U^{2}}[a \ddot{v}(\tau)+U a \dot{\alpha}(\tau) \\
& \left.-b\left(\frac{1}{8}+a^{2}\right) \ddot{\alpha}(\tau)\right]
\end{aligned}
$$

where $L_{a}^{n c}$ and $M_{e a}^{n c}$ denote the non-circulatory parts of lift and moment, respectively.

For a finite-span wing, the modified strip theory as well as the modified lift curve slope can be used to develop the 2-D indicial aerodynamics to $3-\mathrm{D}$. The modified lift curve slope can be expressed as [47]:

$$
\bar{C}_{L_{\alpha}}=C_{L_{\alpha}} \frac{A R}{\sqrt{\left(1-M^{2}\right)} A R+2},
$$

where $A R$ denotes the aspect ratio of the wing.

\subsection{Aeroelastic equation}

After determining the structural and aerodynamic models, one can derive the aeroelastic system through coupling them. Ignoring the drag forces and substituting Eqs. (28) and (29) into the unknown distributed external loads of Eqs. (17) to (19) yield:

$$
\begin{aligned}
& m \ddot{w}+c_{w} \dot{w}+D_{\eta \eta} w^{i v}=G_{w}, \\
& m \ddot{v}-m e_{z} \ddot{\alpha}+c_{v} \dot{v}+D_{\zeta \zeta} v^{i v}=G_{v}+L^{\prime} \\
& j_{\xi} \ddot{\alpha}-m e_{z} \ddot{v}+c_{\alpha} \dot{\phi}-D_{\xi \xi} \alpha^{\prime \prime}=G_{\alpha}+M_{e a}^{\prime} .
\end{aligned}
$$


The system nonlinearities written on the righthand side are caused by large structural deformations and contain inertia, stiffness, damping, and mass imbalance-induced terms up to the third order. The governing equations of motion are often nondimensionalized to make it possible to compare relative values and identify effective parameters dominating the responses of the system. To this end, it is assumed that the cross-section dimensions and material properties of the wing are uniform. Introducing the characteristic length $L$ and the characteristic time $L^{2} \sqrt{m / D_{\zeta}}$, the dependent variables and system parameters are rewritten as follows:

$$
\begin{aligned}
& s^{*}=s / L, \quad b^{*}=b / L, \quad v^{*}=v / L, \\
& w^{*}=w / L, \quad e_{z}^{*}=e / L, \\
& t^{*}=t \sqrt{D_{\zeta} / m L^{4}}, \\
& c_{i}^{*}=c_{i} L^{2} / \sqrt{m D_{\zeta}}, \quad c_{\alpha}^{*}=c_{\alpha} / \sqrt{m D_{\zeta}}, \\
& j_{k}^{*}=j_{k} / m L^{2} \quad \text { where } i=v, w \quad \text { and } \quad k=\xi, \eta, \zeta, \\
& U^{*}=U \sqrt{m L^{2} / D_{\zeta}}, \quad \mu=\pi \rho b^{2} / m, \quad L^{*}=L^{3} L_{a} / D_{\zeta}, \\
& M_{e a}^{*}=L^{2} M_{e a} / D_{\zeta} .
\end{aligned}
$$

Dropping the superscript ' $*$ ' for simplicity, letting $\beta_{\xi} \equiv D_{\xi} / D_{\zeta}$ and $\beta_{\eta} \equiv D_{\eta} / D_{\zeta}$, and using a prime and an overdot to denote non-dimensional spatial and time derivatives, respectively, the nonlinear equations of motion can be expressed in the non-dimensional form as:

$$
\begin{aligned}
& \ddot{w}+c_{w} \dot{w}+\beta_{\eta} w^{i v}=\beta_{\xi}\left(\alpha^{\prime} v^{\prime \prime}\right)^{\prime} \\
& -\beta_{\eta}\left[w^{\prime}\left(w^{\prime} w^{\prime \prime}+v^{\prime} v^{\prime \prime}\right)^{\prime}\right]+\left(\beta_{\eta}-1\right) \\
& {\left[\left(v^{\prime \prime} \alpha+w^{\prime \prime} \alpha^{2}\right)^{\prime}+v^{\prime \prime \prime} \int_{0}^{s} w^{\prime \prime} v^{\prime} d s\right]^{\prime}+j_{\eta} \ddot{w}^{\prime \prime}} \\
& -\frac{1}{2}\left\{w^{\prime} \int_{1}^{s}\left[\int_{0}^{s}\left(v^{\prime 2}+w^{\prime 2}\right)^{\bullet \bullet} d s\right] d s\right\}^{\prime} \\
& -e_{z}\left\{w^{\prime} \int_{1}^{s}\left(w^{\prime}-v^{\prime} \alpha\right)^{\bullet \bullet} d s\right\}^{\prime}-e_{z}\left(v^{\prime} \ddot{v}+w^{\prime} \ddot{w}\right)^{\prime} \\
& +\frac{1}{2} e_{z}\left(v^{\prime 2}+2 w^{\prime 2}+\alpha^{2}-2 \alpha \int_{0}^{s} v^{\prime \prime} w^{\prime} d s\right)^{\bullet},
\end{aligned}
$$

$$
\begin{aligned}
& \ddot{v}-e_{z} \ddot{\alpha}+\left[c_{v}-\frac{\mu C_{L_{\alpha}}}{b \pi} U \phi_{c \alpha}(0)\right] \dot{v} \\
& -\frac{2 \mu C_{L_{\alpha}}}{\pi} U \phi_{c q}(0) \dot{\alpha}-\frac{\mu C_{L_{\alpha}}}{b \pi} U \dot{\phi}_{c \alpha}(0) v \\
& -\frac{\mu C_{L_{\alpha}}}{b \pi} U\left[U \phi_{c \alpha}(0)+2 b \dot{\phi}_{c q}(0)\right] \alpha+v^{i v} \\
& +\frac{\mu C_{L_{\alpha}}}{b \pi} U\left\{v(0) \dot{\phi}_{c \alpha}+2 b \alpha(0) \dot{\phi}_{c q}\right\} \\
& -\frac{\mu C_{L_{\alpha}}}{b \pi} U \sum_{j=1}^{3} \lambda_{v 1 j} B_{1 j}+\frac{\mu C_{L_{\alpha}}}{b \pi} U \sum_{j=1}^{3} \lambda_{\alpha 1 j} B_{2 j} \\
& =-\beta_{\xi}\left(\alpha^{\prime} w^{\prime \prime}\right)^{\prime}-\left[v^{\prime}\left(v^{\prime} v^{\prime \prime}+w^{\prime} w^{\prime \prime}\right)^{\prime}\right]^{\prime} \\
& +\left(\beta_{\eta}-1\right)\left[\left(w^{\prime \prime} \alpha-v^{\prime \prime} \alpha^{2}\right)^{\prime}-w^{\prime \prime \prime} \int_{0}^{s} w^{\prime} v^{\prime \prime} d s\right]^{\prime} \\
& +j_{\zeta} \ddot{v}^{\prime \prime}-\frac{1}{2}\left\{v^{\prime} \int_{1}^{s} \int_{0}^{s}\left({v^{\prime}}^{2}+{w^{\prime}}^{2}\right)^{\bullet \bullet} d s d s\right\}^{\prime} \\
& +e_{z}\left\{\alpha\left[-\frac{1}{2} \int_{0}^{s}\left({v^{\prime}}^{2}+{w^{\prime}}^{2}\right)^{\bullet \bullet} d s+v^{\prime} \ddot{v}+w^{\prime} \ddot{w}\right]\right\}^{\prime} \\
& -e_{z}\left\{v^{\prime} \int_{1}^{s}\left(w^{\prime}-v^{\prime} \alpha\right)^{\bullet \bullet} d s\right\}^{\prime} \\
& -\frac{1}{2} e_{z}\left(v^{\prime 2} \alpha+\frac{1}{3} \alpha^{3}-2 \int_{0}^{s} v^{\prime} w^{\prime \prime} d s\right)^{\bullet \bullet} \\
& j_{\xi} \ddot{\alpha}-e_{z} \ddot{v}+\left[c_{\alpha}-\frac{4 b \mu C_{L_{\alpha}}}{\pi} U \phi_{c m q}(0)\right] \dot{\alpha} \\
& -\frac{2 \mu C_{L_{\alpha}}}{\pi} U \dot{v} \phi_{c m}(0)-\frac{2 \mu C_{L_{\alpha}}}{\pi} U \dot{\phi}_{c m}(0) v \\
& -\frac{2 \mu C_{L_{\alpha}}}{\pi} U\left[U \phi_{c m}(0)+2 b \dot{\phi}_{c m q}(0)\right] \alpha-\beta_{\xi} \alpha^{\prime \prime} \\
& +2 \mu \frac{C_{L_{\alpha}}}{\pi} U\left\{v(0) \dot{\phi}_{c m}+2 b \alpha(0) \dot{\phi}_{c m q}\right\} \\
& -2 \mu \frac{C_{L_{\alpha}}}{\pi} U \sum_{j=1}^{3} \lambda_{v 2 j} B_{1 j}-2 \mu \frac{C_{L_{\alpha}}}{\pi} U \sum_{j=1}^{3} \lambda_{\alpha 2 j} B_{2 j} \\
& =\left(1-\beta_{\eta}\right)\left[\left(v^{\prime \prime 2}-w^{\prime \prime 2}\right) \alpha-v^{\prime \prime} w^{\prime \prime}\right]
\end{aligned}
$$




$$
\begin{aligned}
& +j_{\xi}\left[\int_{0}^{s}\left(v^{\prime \prime} w^{\prime \prime}\right)^{\bullet \bullet} d s-\left(\dot{v}^{\prime} w^{\prime}\right)^{\bullet}\right] \\
& +\left(j_{\eta}-j_{\zeta}\right)\left[\left(\dot{v}^{\prime 2}-\dot{w}^{\prime 2}\right) \alpha-\dot{w}^{\prime} \dot{v}^{\prime}\right] \\
& +e_{z}\left[\frac{1}{2} v^{\prime} \int_{0}^{s}\left(v^{\prime 2}+w^{\prime 2}\right)^{\bullet \bullet} d s-\frac{1}{2}\left(v^{\prime 2}+\alpha^{2}\right) \ddot{v}\right] \\
& +e_{z}\left(\alpha-\int_{0}^{s} v^{\prime \prime} w^{\prime} d s\right) \ddot{w}
\end{aligned}
$$

where the aerodynamic coefficient, $\lambda$, is:

$$
\begin{aligned}
& \lambda_{v 1 j}=b_{j c \alpha}\left(\beta_{j} \frac{U}{b}\right)^{2}, \text { and } \\
& \lambda_{\alpha 1 j}=b \beta_{j}\left(\frac{U}{b}\right)^{2}\left(2 b_{j c q} \beta_{j}-b_{j c \alpha}\right), \\
& \lambda_{v 2 j}=b_{j c m}\left(\beta_{j} \frac{U}{b}\right)^{2}, \text { and } \\
& \lambda_{\alpha 2 j}=b \beta_{j}\left(\frac{U}{b}\right)^{2}\left(2 b_{j c m q} \beta_{j}-b_{j c m}\right) .
\end{aligned}
$$

The resulting aeroelastic equations represent both bending-bending and bending-torsion coupling terms, fully linear and nonlinear contributions of the mass imbalance, and both quadratic and cubic nonlinear terms due to curvature and inertia as well as the unsteady indicial aerodynamic features.

\subsection{Discretizing the equations of motion}

The partial differential Eqs. (42)-(44) are first converted into the corresponding ordinary differential equations using separation of variables and Galerkin's method, and the resulting equations are then expressed in matrix form. Finally, the response analysis can be carried out based on various time domain numerical integration approaches. To this end, it is assumed that the displacement variables can be expressed in the form of a series composed of a linear combination of mode shape functions satisfying the geometric boundary conditions multiplied by time-dependent generalized coordinates as:

$$
\begin{aligned}
& w(s, t)=\sum_{j=1}^{\infty} W_{j}(s) w_{j}(t), \\
& v(s, t)=\sum_{i=1}^{\infty} V_{i}(s) v_{i}(t), \\
& \alpha(s, t)=\sum_{k=1}^{\infty} A_{k}(s) \alpha_{k}(t),
\end{aligned}
$$

where $v_{i}(t), w_{j}(t)$, and $\alpha_{k}(t)$ are generalized coordinates of the system and $V_{i}(s), W_{j}(s)$, and $A_{k}(s)$ are the corresponding mode shape functions, which can be derived from bending, bending, and torsional motions of a cantilever beam, respectively, as follows [48]:

$$
\begin{aligned}
& \Psi_{r}(s)= \cos z_{r} s-\cosh z_{r} s \\
&-\frac{\cos z_{r}+\cosh z_{r}}{\sin z_{r}+\sinh z_{r}}\left(\sin z_{r} s-\sinh z_{r} s\right), \\
& \Psi(s)=W(s) \quad \text { and } \quad V(s), \\
& A_{k}(s)=\sin \left[\frac{1}{2}(2 n-1) \pi s\right] .
\end{aligned}
$$

Recall that $z_{r}$ is a root of the characteristic equation, $1+\cos z \cosh z=0$, and the non-dimensional natural frequencies of vibration of a cantilever beam can be expressed as:

$$
\omega_{v}=z^{2}, \quad \omega_{w}=z^{2} \sqrt{\beta_{\eta}}, \quad \omega_{\alpha}=\frac{1}{2}(2 n-1) \pi \sqrt{\frac{\beta_{\xi}}{j_{\xi}}} .
$$

Substituting the series form of displacements, Eqs. (50) and (51), into the governing equations of motion, Eqs. (42)-(44). and following the Galerkin procedure, one can obtain a set of ordinary differential equations describing the in-plane bending, out-of-plane bending, and torsional motions. In matrix form, these equations may be expressed as:

$$
\begin{aligned}
& {[\mathbf{M}]\{\ddot{\mathbf{q}}\} }+[\mathbf{K}]\{\mathbf{q}\}=\left[\mathbf{M}^{a}\right]\{\ddot{\mathbf{q}}\}+\left[\mathbf{C}^{a}\right]\{\dot{\mathbf{q}}\}+\left[\mathbf{K}^{a}\right]\{\mathbf{q}\} \\
&+\left[\mathbf{M}^{n l}\right]\{\ddot{\mathbf{q}}\}+\left[\mathbf{C}^{n l}\right]\{\dot{\mathbf{q}}\}+\left[\mathbf{K}^{n l}\right]\{\mathbf{q}\} \\
&+[\mathbf{I C}]\{\mathbf{q}(0)\}+[\mathbf{I T}]\{\mathbf{B}\}, \\
& \dot{B}_{i j}^{x}(t)+\left(\frac{U}{b} \beta_{j}\right) B_{i j}^{x}(t)=x_{i}(t), \\
& i=1 \ldots m, \quad j=1 \ldots 3, \text { for } x=v \text { and } \alpha,
\end{aligned}
$$

where $[\mathbf{M}]$ and $[\mathbf{K}]$ are the linear mass and stiffness matrices; $\left[\mathbf{M}^{n l}\right],\left[\mathbf{C}^{n l}\right],\left[\mathbf{K}^{n l}\right]$ and $\left[\mathbf{M}^{a}\right],\left[\mathbf{C}^{a}\right],\left[\mathbf{K}^{a}\right]$ are the nonlinear and aerodynamic matrices, respectively, including mass, stiffness, and damping; and $[\mathbf{I C}]$ and $[\mathbf{I T}]$ are the matrices containing the initial condition and integral terms. The vector of generalized coordinates is defined as:

$$
\begin{aligned}
\{\mathbf{q}(t)\}= & \left\{w_{1}(t), w_{2}(t), \ldots, w_{l}(t), v_{1}(t), v_{2}(t), \ldots,\right. \\
& \left.v_{m}(t), \alpha_{1}(t), \alpha_{2}(t), \ldots, \alpha_{n}(t)\right\}^{T}, \quad(55)
\end{aligned}
$$


and:

$$
\begin{aligned}
& B_{i j}^{x}(t)=\int_{0}^{t} x_{i}(\sigma) e^{-\frac{U}{b} \beta_{j}(t-\sigma)} d \sigma, \\
& x=v \text { and } \alpha .
\end{aligned}
$$

Each of the above matrices is given in the Appendix. It should be noted that some of the matrices involved in Eq. (53) are time-invariant and therefore, they can be evaluated only once, while some other ones are time-variant and should be computed at every time step. Let the state vector be defined as:

$$
\begin{gathered}
\{\mathbf{X}(t)\}=\left\{w_{i}(t), v_{j}(t), \alpha_{k}(t), \dot{w}_{i}(t), \dot{v}_{j}(t),\right. \\
\left.\dot{\alpha}_{k}(t), B_{i p}^{w}(t), B_{j p}^{v}(t), B_{k p}^{\alpha}(t)\right\}^{T}, \\
i=1 \ldots l, \quad j=1 \ldots m, \quad k=1 \ldots n, \quad p=1 \ldots 3,
\end{gathered}
$$

or:

$$
\begin{aligned}
& \{\mathbf{X}(t)\}= \\
& \quad\left\{\{\mathbf{q}(t)\}^{T},\{\dot{\mathbf{q}}(t)\}^{T}, B_{i p}^{w}(t), B_{j p}^{v}(t), B_{k p}^{\alpha}(t)\right\}^{T} \\
& i=1 \ldots l, \quad j=1 \ldots m, \quad k=1 \ldots n, \quad p=1 \ldots 3,
\end{aligned}
$$

where $l, m$ and $n$ represent the numbers of in-plane, out-of-plane, and torsional modes, respectively.

Similarly to the conventional process of converting two-dimensional differential equations to a set of coupled first-order differential equations, using the concept of the state space formulation, the governing equations of motion Eqs. (53) and (54) could be rewritten as

$$
\begin{aligned}
& {\left[\mathbf{M}^{T V}(t)\right]\{\dot{\mathbf{X}}(t)\}=\left[\mathbf{K C}^{T V}(t)\right]\{\mathbf{X}(t)\}} \\
& +\left[\mathbf{I C}^{T I}(t)\right]\{\mathbf{X}(0)\}
\end{aligned}
$$

where the time-variant matrices $\left[\mathbf{M}^{T V}(t)\right]$ and
$\left[\mathbf{K C}^{T V}(t)\right]$ along with the time-invariant initial condition matrix $\left[\mathbf{I C}^{T I}\right]$ are squares on the order of and can be assembled by Eq. (59) as shown in Box II, where $\sum=l+m+n$ represents the total number of modes and [I] and [0] denote the unit and zero matrices, respectively. It is noteworthy that the matrix denoted by $[\mathbf{B}]$ should be constructed using the terms on the right-hand side of Eq. (54). Now, the firstorder ordinary differential equations can be solved numerically by applying the standard Runge-Kutta time-marching scheme.

\section{Results and discussion}

To investigate the nonlinear aeroelastic behavior of an HAR wing in the subsonic compressible flow, the linear eigenvalue and response analyses are performed in the time domain. For this purpose, consider two wing models with different geometric and material properties: the modified Daedalus Human Powered Aircraft (HPA) [3] and the Goland Wing (GW) [49], as shown in Table 3 . The first one is usually used to model the highly flexible wings such as those utilized in HALE aircrafts.

Validity of the method developed here can be confirmed through comparing the aeroelastic behaviors of the HPA wing, including the flutter boundary and time responses in the incompressible flight speed regime, with those that are available in $[3,30]$. The same unsteady aerodynamics as those in the aforementioned resources are obtained using both circulatory and non-circulatory parts of aerodynamic lift and moment described by Eqs. (28)-(29) and Eqs. (35)-(36), respectively, for which the approximating coefficients are taken from Tables 1 and 2 for the Mach number of zero. Using the eigenvalue or the time response approach and the HPA wing characteristics described in Table 3 , the linear flutter boundary can be determined through the governing aeroelastic equations given by $\mathrm{Eq}$. (58). It

$$
\begin{aligned}
& \mathbf{M}^{T V}(t)=\left[\begin{array}{ccc}
{[\mathbf{I}]_{\Sigma \times \Sigma}} & {[0]_{\Sigma \times \Sigma}} & {[0]_{\Sigma \times 3 \Sigma}} \\
{[0]_{\Sigma \times \Sigma}} & {\left[\mathbf{M}^{l}-\mathbf{M}^{a}-\mathbf{M}^{n l}\right]_{\Sigma \times \Sigma}} & {[0]_{\Sigma \times 3 \Sigma}} \\
{[0]_{3 \Sigma \times \Sigma}} & {[0]_{3 \Sigma \times \Sigma}} & {[\mathbf{I}]_{3 \Sigma \times 3 \Sigma}}
\end{array}\right], \\
& \mathbf{K C}^{T V}(t)=\left[\begin{array}{ccc}
{[0]_{\Sigma \times \Sigma}} & {[\mathbf{I}]_{\Sigma \times \Sigma}} & {[0]_{\Sigma \times 3 \Sigma}} \\
-\left[\mathbf{K}^{l}-\mathbf{K}^{n l}-\mathbf{K}^{a}\right]_{\Sigma \times \Sigma} & {\left[\mathbf{C}^{n l}+\mathbf{C}^{a}\right]_{\Sigma \times \Sigma}} & {[\mathbf{I T}]_{\Sigma \times 3 \Sigma}} \\
{[\mathbf{B}]_{3 \Sigma \times \Sigma}} & {[0]_{3 \Sigma \times \Sigma}} & -\frac{U}{b}[\boldsymbol{\beta}]_{3 \Sigma \times 3 \Sigma}
\end{array}\right], \\
& \mathbf{I C}^{T I}(t)=\left[\begin{array}{ccc}
{[0]_{\Sigma \times \Sigma}} & {[0]_{\Sigma \times \Sigma}} & {[0]_{\Sigma \times 3 \Sigma}} \\
{[\mathbf{I C C}]_{\Sigma \times \Sigma}} & {[0]_{\Sigma \times \Sigma}} & {[0]_{\Sigma \times 3 \Sigma}} \\
{[0]_{3 \Sigma \times \Sigma}} & {[0]_{3 \Sigma \times \Sigma}} & {[0]_{3 \Sigma \times 3 \Sigma}}
\end{array}\right]
\end{aligned}
$$


Table 3. Wing model data.

\begin{tabular}{lll}
\hline Wing property & HPA & GW \\
\hline Half-span (m) & 16 & 6.096 \\
Chord (m) & 1 & 1.829 \\
Mass per unit length (kg.m) & 0.75 & 35.695 \\
Moment of inertia (kg.m) & 0.1 , about mid-chord & 8.694 , about cg \\
Span-wise elastic axis (m) & mid-chord & 0.6096, from leading edge \\
Center of gravity (m) & mid-chord & 0.7925, from leading edge \\
Bending rigidity (N.m $\left.{ }^{2}\right)$ & $2 \times 10^{4}$ & $9.765 \times 10^{6}$ \\
Torsional rigidity (N.m $\left.{ }^{2}\right)$ & $1 \times 10^{4}$ & $0.989 \times 10^{6}$ \\
Chordwise bending rigidity $\left(\mathrm{N} . \mathrm{m}^{2}\right)$ & $4 \times 10^{6}$ & $9.765 \times 10^{8}$ \\
Flight condition: & & $8 \mathrm{~km}$ \\
Altitude & $20 \mathrm{~km}$ & $0.525 \mathrm{~kg} \cdot \mathrm{m}^{3}$ \\
Density of air & $0.0889 \mathrm{~kg} \cdot \mathrm{m}^{3}$ & \\
\hline
\end{tabular}

Table 4. Linear flutter and nonlinear Limit Cycle Oscillation (LCO) boundary of Human Powered Aircraft (HPA) wing, comparison of the present results with those of $[3,30]$.

\begin{tabular}{lcccc}
\hline & Initial disturbance $\boldsymbol{v}_{\mathbf{0}}(\mathbf{m})$ & Present & Reference [3] & Reference [30] \\
\hline Flutter speed, $U_{f}(\mathrm{~m} / \mathrm{s})$ & - & 32.60 & 32.21 & 32.71 \\
Flutter frequency, $\omega_{f}(\mathrm{rad} / \mathrm{s})$ & - & 22.39 & 22.61 & 22.05 \\
LCO boundary, speed $(\mathrm{m} / \mathrm{s})$ & 0.02 & 32.60 & 32.20 & 32.71 \\
LCO boundary, frequency $(\mathrm{rad} / \mathrm{s})$ & 0.02 & 22.38 & 22.61 & 22.05 \\
LCO boundary, speed $(\mathrm{m} / \mathrm{s})$ & 1 & 32.31 & $\cong 32$ & 31.94 \\
LCO boundary, speed $(\mathrm{m} / \mathrm{s})$ & 2 & 30.55 & $\cong 30$ & 30.00 \\
LCO boundary, speed $(\mathrm{m} / \mathrm{s})$ & 4 & 28.33 & $\cong 28$ & 28.13 \\
\hline
\end{tabular}

should be noted that due to the decreasing effects of the higher-mode contributions to the results [30], the first two modes in each degree of freedom are considered to investigate the interaction among the motions and describe the response. Moreover, the eigenvalue analysis of bending-bending-torsion equations is converted into that of bending-torsion ones by eliminating nonlinear coupling terms and ignoring the effect of drag force.

The linear flutter and nonlinear LCO boundaries, speed, and frequency at which the LCO is initiated for various ITDs are shown in Table 4 based on the present study and those reported in [3,30]. It can be observed that there is good agreement between the results.

For further comparison, system responses at the flight speed of about $28 \mathrm{~m} / \mathrm{s}$ for various initial disturbances are shown in Figure 5 . It can be seen that the wing tip response almost tends to identical LCO oscillating with a mean of around $1.5 \mathrm{~m}$ for the initial tip deflection of $4 \mathrm{~m}$, whereas it settles down to the stable equilibrium state with the initial deflection of less than $1 \mathrm{~m}$. These results are quantitatively and qualitatively in good agreement with those reported in the aforementioned references and hence, accuracy

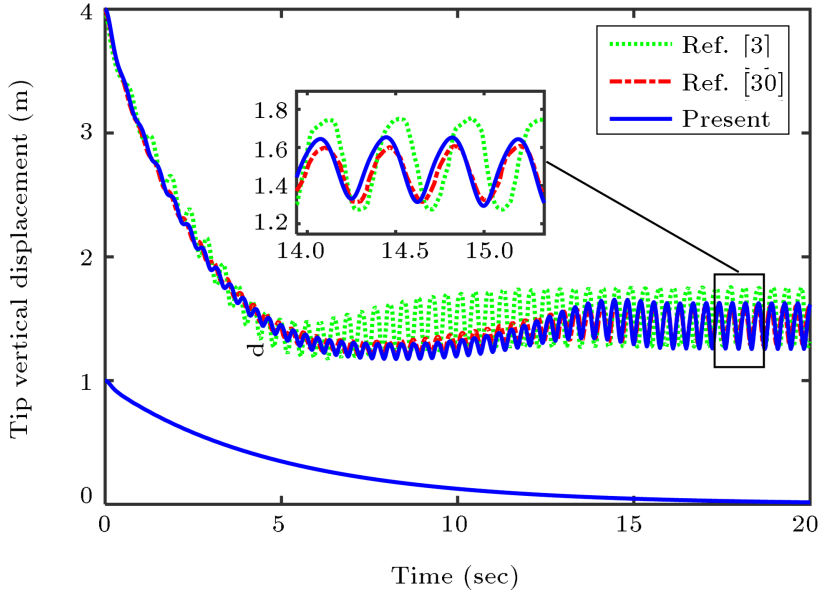

Figure 5. Comparison of the nonlinear tip vertical displacements between the present study and references [3, 30] for an initial disturbance of $4 \mathrm{~m}$ at the velocity of about $28 \mathrm{~m} / \mathrm{sec}$.

and validity of the unsteady indicial aerodynamics, nonlinear structural modelling, and consequently the developed nonlinear aeroelastic model are confirmed. Note that the differences, though small, may be due to 
the different aerodynamic models, the way of Wagner function approximation, i.e. the four indicial functions describing the unsteady aerodynamics in incompressible flow instead of single Wagner function, the solution approach, and the nonlinear terms which have been taken into account.

Analysis of the results indicates that an LCO may exist below the linear flutter speed if sufficient (critical) disturbance is applied to the system. Bellow this critical value, the wing returns to its initial equilibrium state and beyond it, an identical LCO seems to be experienced by the wing. Such a behavior can be classified in detrimental nonlinearity [50]. Moreover, at small oscillation amplitudes, the in-plane bending modes seem not to be excited and therefore, the response of the system, which oscillates about tip displacement values near zero, may be determined using aeroelastic equations describing out-of-plane bending and torsional motions. However, the in-plane modes considerably affect the behavior of the system as the amplitude of oscillations increases. In fact, strong nonlinear coupling between chord-wise bending and torsion causes a significant jump in the mean state of oscillations [3]. Consequently, ignoring the in-plane motion in such wings could result in not identifying the oscillations about states, except for the zero deflection equilibrium state. On the other hand, the number of modes being considered in spatial discretization could affect the aeroelastic response behavior. The effect of the number of modes on tip vertical displacement is shown in Figure 6 at the velocity of $30.6 \mathrm{~m} / \mathrm{sec}$ for

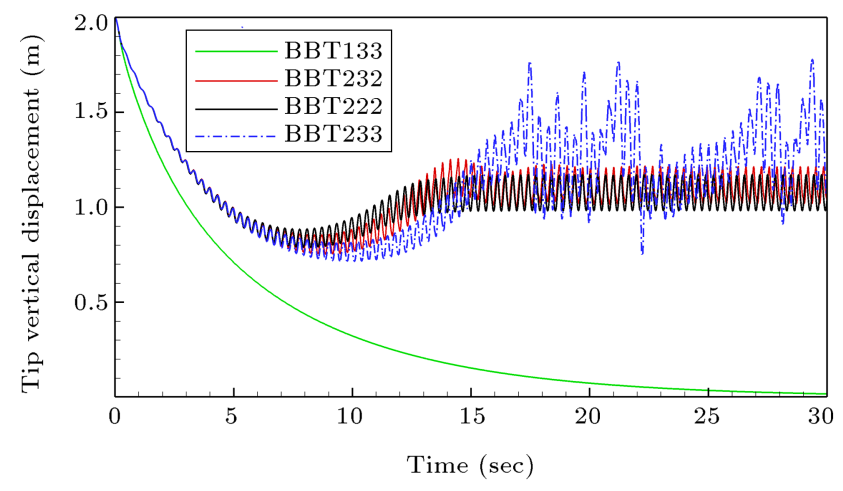

Figure 6. Effect of the number of modes included in the analysis of tip vertical displacement at the velocity of 30.6 $\mathrm{m} / \mathrm{sec}$ for an initial disturbance of $2 \mathrm{~m}$. an initial disturbance of $2 \mathrm{~m}$. Note that BBT denotes out-of-plane bending-in-plane bending-torsion motion analysis and each digit of the number denotes the number of the corresponding mode being taken into account. It can be seen that the wing tip displacement tends to the same LCO in BBT222 and BBT232 cases, whereas for BBT133, it cannot accumulate enough energy to jump to an LCO and therefore, it returns to the stable equilibrium state. For the BBT233 case, the response loses its periodicity and becomes aperiodic (chaotic). It should be noted that the two latter cases indicate the system response before and after initiation of the LCO at the given speed, that is, one can obtain the same LCO as shown in Figure 6 by increasing and decreasing the speed, respectively.

It should be noted that the aeroelastic analyses generally involve the low-frequency modes, which have the main contribution in the occurrence of critical phenomena such as flutter and LCO. In this study, a linear modal analysis has been carried out to determine natural frequencies of the wing for all three components of motion including out of plane, in-plane, and torsion. As it is shown in Table 5, the in-plane modes have much higher frequencies than the other two components due to higher in-plane stiffness, especially in GW model. Therefore, it would be reasonable not to take the second and higher modes into account in areoelastic investigations.

To investigate the aeroelastic behavior of HAR wings experiencing speeds greater than Mach 0.3, compressibility effects should be considered. The GW model with the characteristics given in Table 3 has a dynamic aeroelastic instability in the compressible range and hence, it has been chosen as the second case of study. The linear flutter boundary of GW in the incompressible flow regime reported in several papers [49,51-53] along with the calculated values in the present study is shown in Table 6, indicating a very good agreement between the results. Note that, as mentioned earlier, the results are same as those of bending-torsion analysis. The values given in Table 6 show that the flutter speed occurs at subsonic compressible flow regime. To provide a reasonable description of the aeroelastic analysis, therefore, appropriate Mach-dependent aerodynamic loads should be taken into account. The approximating coefficients of the indicial functions associated with flight Mach

Table 5. Natural frequencies $(\mathrm{Hz})$ of uncoupled in-plane bending, out-of-plane bending, and torsion for benchmark wing models.

\begin{tabular}{ccccccc}
\hline Mode & In-plane & $\begin{array}{c}\text { HPA } \\
\text { out-of-plane }\end{array}$ & Torsion & In-plane & $\begin{array}{c}\text { GW } \\
\text { out-of-plane }\end{array}$ & Torsion \\
\hline 1 & 5.048 & 0.357 & 4.941 & 78.761 & 7.876 & 13.832 \\
2 & 31.636 & 2.237 & 14.823 & 493.589 & 49.359 & 41.496 \\
3 & 88.582 & 6.264 & 24.705 & 1382.064 & 138.206 & 69.160 \\
\hline
\end{tabular}


Table 6. Flutter speed and frequency of Goland Wing (GW) model according to various references and the present study.

\begin{tabular}{lcccc}
\hline Reference & $\begin{array}{c}\text { Flutter speed } \\
(\mathbf{m} / \mathbf{s})\end{array}$ & $\begin{array}{c}\text { Error } \\
\mathbf{( \% )}\end{array}$ & $\begin{array}{c}\text { Flutter frequency } \\
(\mathbf{r a d} / \mathbf{s})\end{array}$ & $\begin{array}{c}\text { Error } \\
(\mathbf{\%})\end{array}$ \\
\hline Goland [51] (Exact) & 137.25 & - & 70.67 & - \\
Gern and Librescu [52] & 137.11 & 0.10 & 75.52 & 6.82 \\
Patil et al. [53] & 137.26 & 0.00 & 70.18 & 0.71 \\
Fazelzadeh et al. [49] & 137.01 & 0.17 & 69.93 & 1.07 \\
Present & 137.04 & 0.15 & 69.93 & 1.05 \\
\hline
\end{tabular}

number could be taken from the curves shown in Figure 4. Considering the point mentioned about virtual mass effect in the compressible unsteady aerodynamic theory, the aerodynamic loads are obtained from Eqs. (28) and (29).

Now, dynamic instability and aeroelastic time responses of GW in subsonic compressible region are investigated by Eigen-analysis and time integration of the governing first-order ordinary differential equations (Eq. (58)). Note that since lift curve slope and air density are functions of flight conditions such as free stream Mach number and altitude, they should be modified through variation of flight conditions. On the other hand, considering the dependency of aerodynamic loads on Mach number in the compressible flow, the flutter boundary has to be determined through an iterative process using the appropriate indicial functions. To investigate the effect and importance of compressibility, the LCO boundary of the GW model (i.e. the speed and frequency in which LCO occurs) is determined in different flight conditions. A comparison of the results is given in Figure 7 for both the incompressible and compressible cases.

The results indicate an increasing difference in LCO boundaries evaluated in both cases as the Mach number increases. It can be seen that for the GW model, this increasing difference may reach up to 12 percent in the considered flight conditions in this

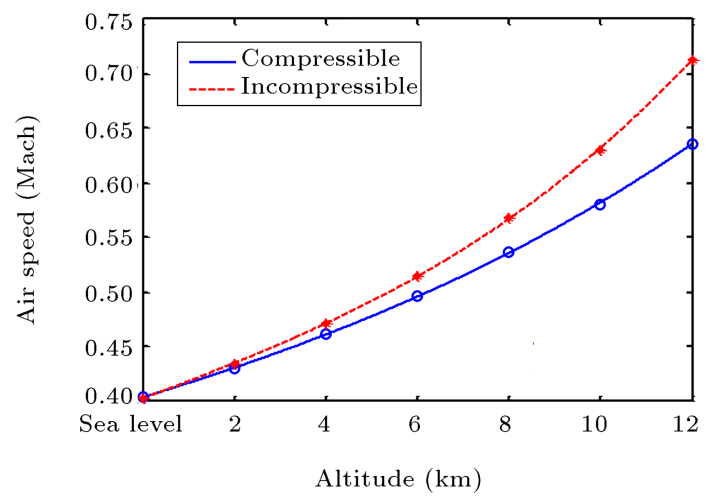

(a) study. Thus, considering the compressibility effects is required to achieve a more realistic model of the behavior of an HAR wing. As shown in Figure 7, flight at higher altitudes increases the speed by which an LCO initiated. Moreover, the flight conditions such as altitude and Mach number affect the amplitude of an LCO. In this regard, for the compressible case, as shown in Figure 8, it can be said that the amplitude of an LCO experiences an increase at higher flight speeds,

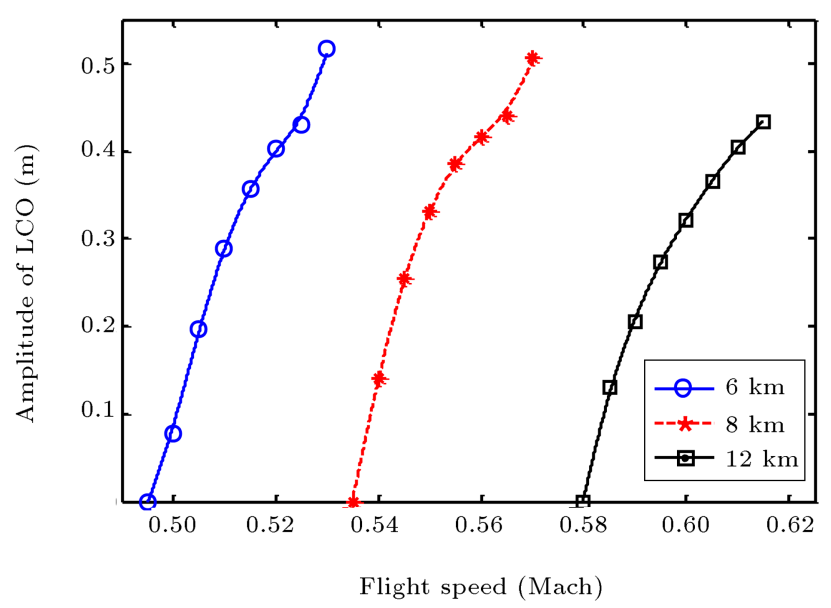

Figure 8. Amplitude of the Limit Cycle Oscillation (LCO) for various flight conditions in the compressible flight speed regime.

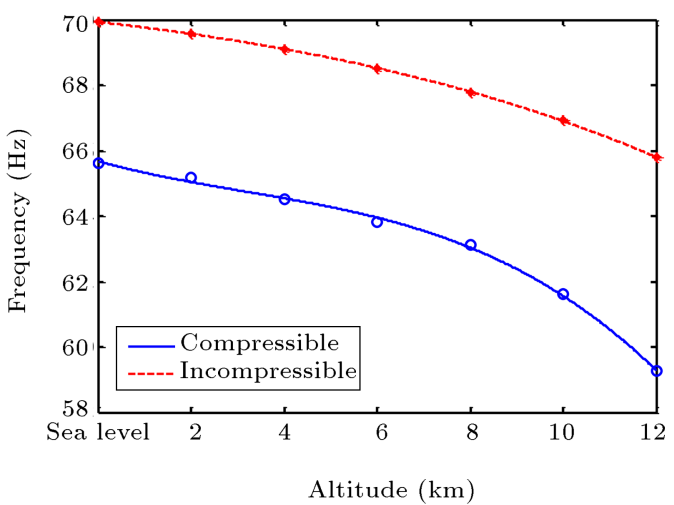

(b)

Figure 7. Limit Cycle Oscillation (LCO) boundary of the Goland Wing (GW) model at given altitudes for both compressible and incompressible flows: (a) Air speed, and (b) frequency of LCO. 


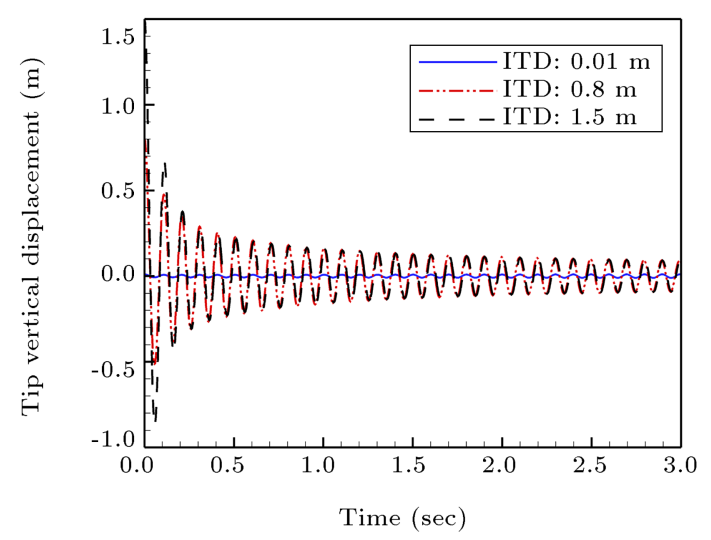

(a)

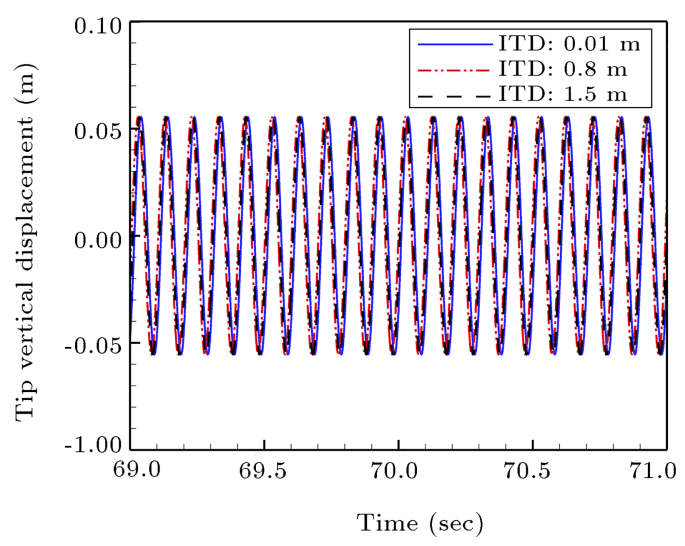

(b)

Figure 9. Effect of Initial Tip Disturbances (ITDs) on tip vertical displacement of the Goland Wing (GW) model at the velocity of $183 \mathrm{~m} / \mathrm{s}$ : (a) Transient response and (b) steady-state response.

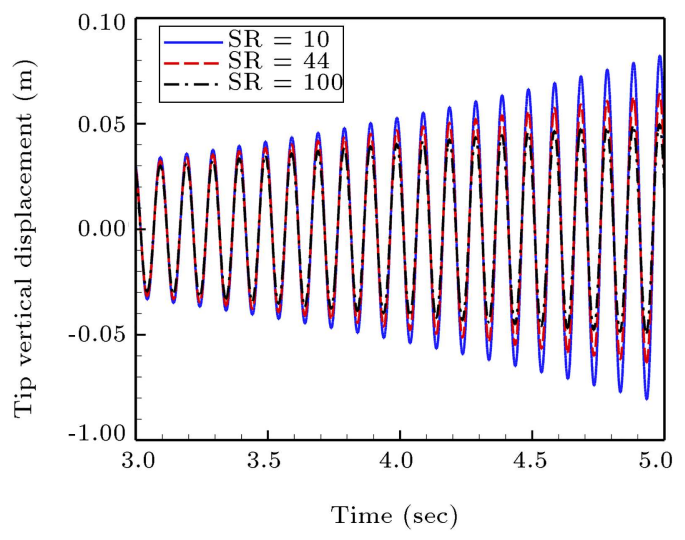

(a)

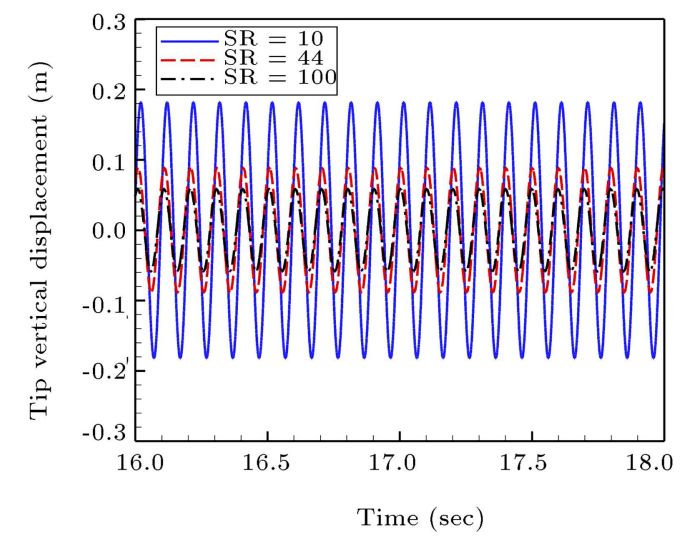

(b)

Figure 10. Tip vertical response of the Goland wing at the flight condition with the Mach of 0.4 and altitude of 8 km for various Stiffness Ratios (SRs): (a) Transient response and (b) steady-state response.

whereas it can significantly decrease through increase in the flight altitude.

It should be noted that, contrarily to the HPA model in which LCOs are expected to begin before the linear flutter speed for some initial disturbances, the GW model has almost the same linear flutter and LCO boundaries. To investigate the initial conditions for the aeroelastic behavior of the GW model, the time responses of such a model at the flight condition given in Table 3 to various ITDs are shown in Figure 9. Despite oscillations with different amplitudes in the transient part of response, they go into identical LCO with the same amplitude and frequency in the steadystate response, which means that the initial disturbances cannot change the characteristics of LCO. The $\mathrm{SR}$ between the out-of-plane and in-plane bending motions, i.e. $\beta_{\eta}$, plays an important role in the dynamics of a nonlinear system [4]. Figure 10 shows the SR effect on the post-flutter response of the GW model at the flight condition given in Table 3 . It can be observed that the amplitude of LCO decreases with increase in the SR and it reaches its minimum value when the outof-plane mode effect can be ignored due to high rigidity.

Now, the linear and nonlinear time responses of the GW model are to be investigated for the specific flight condition with initial disturbances of $0.1 \mathrm{~m}$ and 3 degrees in tip vertical displacement and twist, respectively. Note that, as there are linear and nonlinear coupling terms in the governing aeroelastic equation of motions, all the modes are excited by each of the aforementioned initial conditions. The flutter and postflutter time responses along with their corresponding phase plane diagrams at the flight speeds of 197.5 and $200 \mathrm{~m} / \mathrm{s}$, respectively, and altitude of $10 \mathrm{~km}$ are shown in Figures 11 and 12(a). Although the linear model shows constant and exponentially increasing amplitude oscillations at the flutter and post-flutter speeds, respectively, a nonlinear mechanism, as expected, limits the linear response to a stable LCO about the initial equilibrium state. It can be inferred that there are two stable attractors in the nonlinear GW model, a fixed point and a limit cycle, which are distinguished 


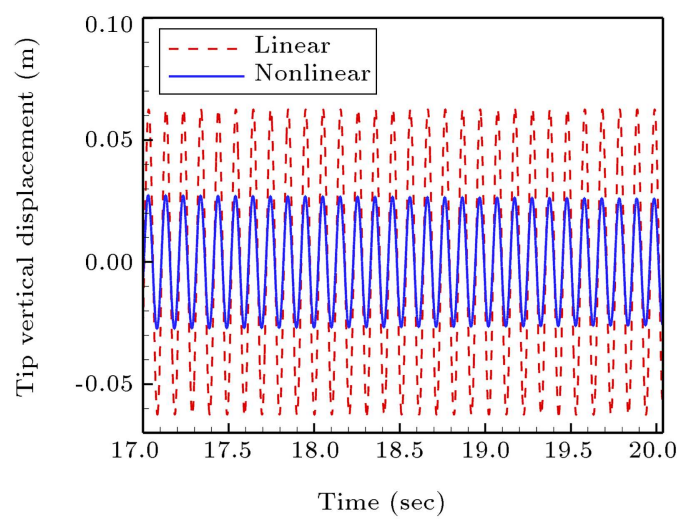

(a)

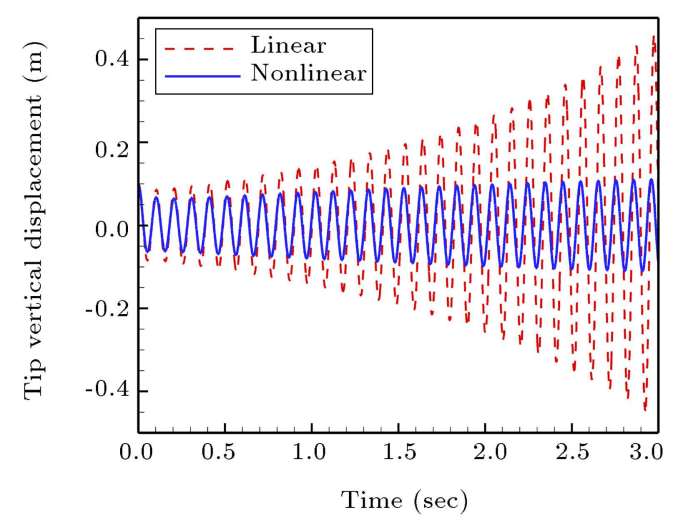

(b)

Figure 11. The linear and nonlinear time responses of the Goland Wing (GW) model to initial disturbances of $v_{0}=0.1 \mathrm{~m}$ and $\alpha_{0}=3^{\circ}$ at the flight altitude of $10 \mathrm{~km}$ : (a) Flutter speed of 197.5 and (b) post-flutter speed of $200 \mathrm{~m} / \mathrm{s}$.

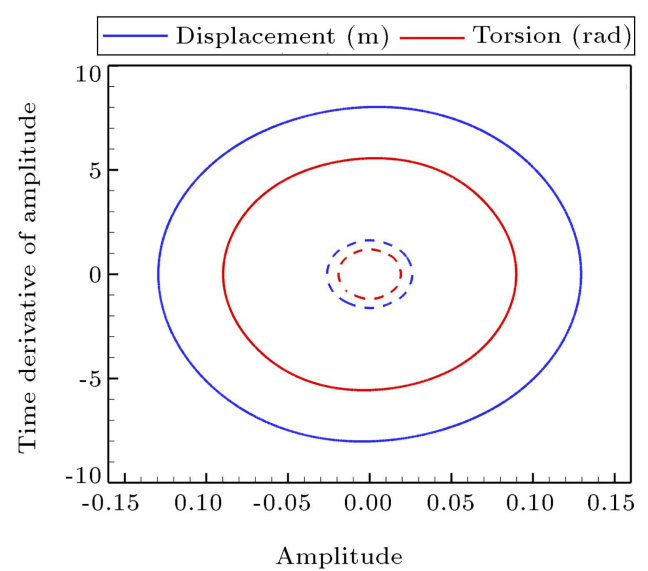

(a)

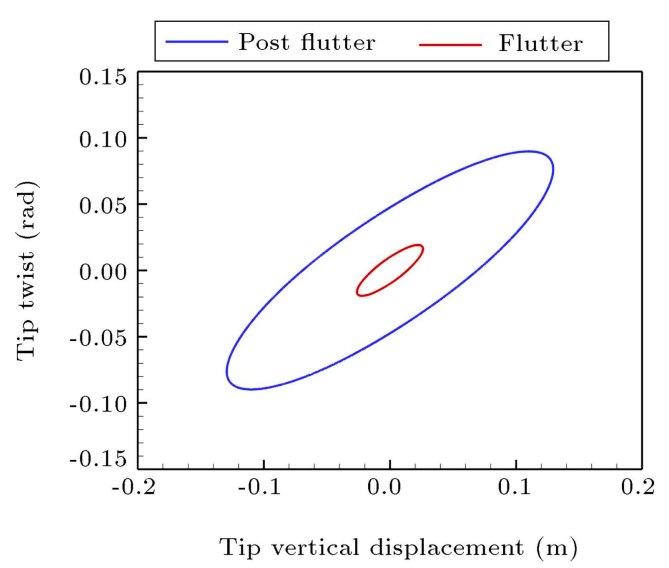

(b)

Figure 12. Phase plane plots at the flutter and post-flutter speeds of $197.5 \mathrm{~m} / \mathrm{s}$ (dashed) and $200 \mathrm{~m} / \mathrm{s}$ (solid), respectively: (a) $v$ and $\alpha$ versus $\dot{v}$ and $\dot{\alpha}$ and (b) tip vertical displacement versus tip twist.

from each other by an unstable LCO at the linear flutter speed. In other words, the wing tip oscillations can settle down in an equilibrium and the specific periodic state below and beyond the linear flutter speed, respectively, with various initial disturbances of $v_{0}$ and $\alpha_{0}$. It is noteworthy that, contrarily to the HPA wing model, this system has no LCO below the linear flutter speed and hence, its dynamic behavior falls into the benign nonlinearity category [50]. Figure 2(b) presents the phase plane plot of LCO describing tip vertical displacement versus tip twist at the post-flutter speed of $200 \mathrm{~m} / \mathrm{s}$. The ellipsoid form of phase plane diagrams indicates that the oscillations are sinusoidal. Following the investigation into various parameters of the dynamic behavior of the GW, the effects of nonlinearity due to inertia and mass imbalance have also been studied in the present work. The results indicate the negligible contribution of these terms to the dynamic response of the system. In other words, the structural nonlinearities have a dominant effect on overall nonlinearity in such a wing model (Figure 13).

\section{Concluding remarks}

Nonlinear aeroelastic behavior of High-Aspect-Ratio (HAR) wings undergoing large deflection in the unsteady subsonic flow regime was considered in the present study. Hence, adopting the nonlinear beam theory and the unsteady indicial aerodynamics, a nonlinear formulation describing dynamic response of HAR wings was first derived valid over the entire range of subsonic flow, i.e. $0 \leq M \leq 0.8$. The developed nonlinear structural model included the in-plane and out-ofplane bending and torsional motions, all nonlinearities up to cubic order arising from large deformation, mass distribution, and mass imbalance at the cross section of the wing. On the other hand, comprehensive and efficient unsteady aerodynamic loads based on indicial functions were introduced. Unique representation of the indicial functions about any arbitrary axis of rotation, unified aerodynamic formulations for both incompressible and subsonic compressible flows, appropriate form of unsteady aerodynamic loads to 


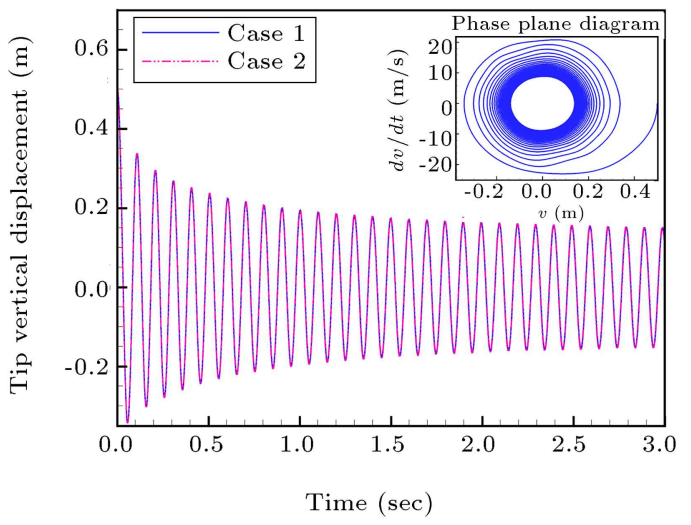

(a)

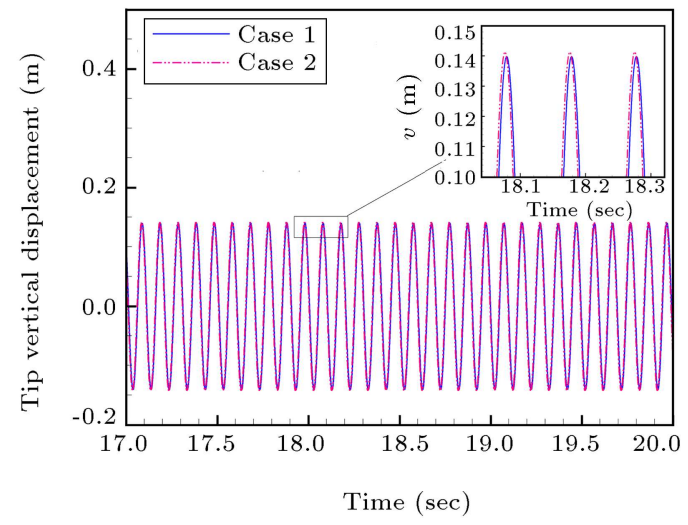

(b)

Figure 13. Tip vertical response of the Goland wing at the flight condition with the Mach of 0.4 and altitude of $8 \mathrm{~km}$. Case 1: all nonlinear terms have been considered; Case 2: nonlinearities due to inertia and mass imbalance have been ignored: (a) Transient response and (b) steady-state response.

be applied to the structural model, and reduction in the complexity of governing aeroelastic equations and consequently, in the computational cost of time integration in the analysis of nonlinear aeroelastic behavior were some advantages of the applied method. The verification process was carried out through comparing the dynamic behaviors of two benchmark wing models in the present study and those reported in other references. Good agreement was obtained, which confirmed the nonlinear structural, indicial aerodynamic, and consequently nonlinear aeroelastic modelling.

Another aim of the present work was to investigate the effect of various parameters including the wing characteristics, initial disturbances, and flight conditions on the nonlinear dynamic response of the system. It was shown that compressibility could affect the Limit Cycle Oscillation (LCO) boundary up to 12 percent (over the range of flight speed studied here) in comparison with the incompressible case. That is, appropriate Mach-dependent aerodynamics could lead to a more reasonable and realistic description of the dynamic behavior of the system. Beyond the linear flutter speed, in both cases of study, almost the same LCOs were observed for various Initial Tip Disturbances (ITDs). However, under sufficiently large disturbances, as it was shown in the Human Powered Aircraft (HPA) wing model analysis, one could detect an LCO below the linear flutter speed, whereas such behavior was not observed in the Goland Wing (GW) model. Amplitude of the LCOs decreased with increase in the magnitude of Stiffness Ratio (SR) up to its minimum in the condition in which the out-of-plane mode effect could be ignored due to its high rigidity.

\section{References}

1. Xiang, J., Yan, Y., and Li, D. "Recent advance in nonlinear aeroelastic analysis and control of the aircraft", Chinese Journal of Aeronautics, 27(1), pp. 12-22 (2014). DOI: 10.1016/j.cja.2013.12.009

2. Nayfeh, A.H. and Pai, P.F., Linear and Nonlinear Structural Mechanics, pp. 171-266, John Wiley \& Sons, Germany (2004).

3. Patil, M.J., Hodges, D.H., and Cesnik, C.E. "Limitcycle oscillations in high-aspect-ratio wings", Journal of Fluids and Structures, 15(1), pp. 107-132 (2001). DOI: $10.1006 /$ jlls.2000.0329

4. Strganac, T., Cizmas, P., Nichkawde, C., et al. "Aeroelastic analysis for future air vehicle concepts using a fully nonlinear methodology", 46th AIA A/ASME/ASCE/AHS/ASC Structures, Structural Dynamics and Materials Conference, Austin, Texas (2005).

5. Hodges, D.H. and Dowell, E. "Nonlinear equations of motion for the elastic bending and torsion of twisted nonuniform rotor blades", NASA TN D-7818 (1974).

6. Dowell, E., Traybar, J., and Hodges, D.H. "An experimental-theoretical correlation study of nonlinear bending and torsion deformations of a cantilever beam", Journal of Sound and Vibration, 50(4), pp. 533-544 (1977). DOI: 10.1016/0022-460X(77)90501-6

7. Rosen, A. and Friedmann, P. "The nonlinear behavior of elastic slender straight beams undergoing small strains and moderate rotations", Journal of Applied Mechanics, 46(1), pp. 161-168 (1979). DOI: 10.1115/1.3424490

8. Crespo da Silva, M. and Glynn, C. "Nonlinear flexural-flexural-torsional dynamics of inextensional beams. I. Equations of motion", Journal of Structural Mechanics, 6(4), pp. 437-448 (1978). DOI: 10.1080/03601217808907348

9. Pai, P.F. and Nayfeh, A. H. "Three-dimensional nonlinear vibrations of composite beams-I. Equations of motion", Nonlinear Dynamics, 1(6), pp. 477-502 (1990). DOI: $10.1007 / \mathrm{BF} 01856950$ 
10. Nayfeh, A.H. and Pai, P.F. "Non-linear non-planar parametric responses of an inextensional beam", International Journal of Non-Linear Mechanics, 24(2), pp. 139-158 (1989). DOI: 10.1016/0020-7462(89)90005-X

11. Hodges, D.H. "A mixed variational formulation based on exact intrinsic equations for dynamics of moving beams", International Journal of Solids and Structures, 26(11), pp. 1253-1273 (1990).

12. Bakhtiarinezhad, F. and Shokrollahi, S. "Threedimensional eigenmode flutter analysis of a rectangular cantilever plate in low subsonic flow", Scientia Iranica, 11(1-2), pp. 60-68 (2004).

13. Katz, J. and Plotkin, A., Low-speed Aerodynamics, 13, Cambridge university press (2001).

14. Kier, T.M. "Comparison of unsteady aerodynamic modelling methodologies with respect to flight loads analysis", In Proceedings of the AIAA Atmospheric Flight Mechanics Conference and Exhibit (2005).

15. Leishman, J. "Validation of approximate indicial aerodynamic functions for two-dimensional subsonic flow", Journal of Aircraft, 25(10), pp. 914-922 (1988). DOI: $10.2514 / 3.45680$

16. Marzocca, P., Librescu, L., and Chiocchia, G. "Unsteady aerodynamics in various flight speed regimes for flutter/dynamic response analyses", In 18th AIA A Applied Aerodynamic Conference, AIAA 2000-4229, Denver, CO, pp. 491-501 (2000).

17. Murua, J., Palacios, R., and Graham, J.M.R. "Applications of the unsteady vortex-lattice method in aircraft aeroelasticity and flight dynamics", Progress in Aerospace Sciences, 55, pp. 46-72 (2012).

18. Mazelsky, B. "Numerical determination of indicial lift of a two-dimensional sinking airfoil at subsonic Mach numbers from oscillatory lift coefficients with calculations for Mach number 0.7", NACA TN 2562 (1951).

19. Mazelsky, B. "Determination of indicial lift and moment of a two-dimensional pitching airfoil at subsonic Mach numbers from oscillatory coefficients with numerical calculations for a Mach number of 0.7 ", NACA TN 2613 (1952).

20. Mazelsky, B. and Drischler, J.A. "Numerical determination of indicial lift and moment functions for a two-dimensional sinking and pitching airfoil at Mach numbers 0.5 and 0.6", NACA TN 2739 (1952).

21. Marzocca, P., Librescu, L., and Chiocchia, G. "Aeroelastic response of a $2-\mathrm{D}$ airfoil in a compressible flow field and exposed to blast loading", Aerospace Science and Technology, 6(4), pp. 259-272 (2002). DOI: 10.1016/S1270-9638(02)01169-0

22. Marzocca, P., Librescu, L., Kim, D., et al. "Development of an indicial function approach for the two-dimensional incompressible/compressible aerodynamic load modelling", Proceedings of the Institution of Mechanical Engineers, Part G: Journal of Aerospace Engineering, 221(3), pp. 453-463 (2007). DOI: $10.1243 / 09544100 J A E R O 88$
23. Farsadi, T. and Javanshir, J. "Expansion of indicial function approximations for 2-D subsonic compressible aerodynamic loads", In Proceedings of the ASME 2012 International Mechanical Engineering Congress and Exposition, Houstoun, Texas, pp. 519-528 (2012).

24. Nejati, M., Shokrollahi, S., and Shams, S. "A comprehensive model to compute incompressible-subsonic compressible unsteady aerodynamic loads using indicial functions", Modares Mechanical Engineering, 17(1), pp. 353-364 (in Persian) (2017).

25. Nejati, M., Shokrollahi, S., and Shams, S. "Nonlinear aeroelastic analysis of high-aspect-ratio wings using indicial aerodynamics", Journal of the Brazilian Society of Mechanical Sciences and Engineering, 40(6), p. 298 (2018).

26. Bisplinghoff, R.L., Ashley, H., and Halfman, R.L., Aeroelasticity, pp. 323-353, Dover Publications, New York (1996).

27. Qin, Z. and Librescu, L. "Aeroelastic instability of aircraft wings modelled as anisotropic composite thinwalled beams in incompressible flow", Journal of Fluids and Structures, 18(1), pp. 43-61 (2003). DOI: 10.1016/S0889-9746(03)00082-3

28. Tang, D. and Dowell, E.H. "Experimental and theoretical study on aeroelastic response of high-aspect-ratio wings", AIA A Journal, 39(8), pp. 1430-1441 (2001). DOI: $10.2514 / 2.1484$

29. Shams, S., Lahidjani, M.S., and Haddadpour, H. "Nonlinear aeroelastic response of slender wings based on Wagner function", Thin-Walled Structures, 46(11), pp. 1192-1203 (2008). DOI: 10.1016/j.tws.2008.03.001

30. Shams, S., Sadr, M., and Haddadpour, H. "An efficient method for nonlinear aeroelasticy of slender wings", Nonlinear Dynamics, 67(1), pp. 659-681 (2012). DOI: 10.1007/s11071-011-0018-2

31. Jian, Z. and Jinwu, X. "Nonlinear aeroelastic response of high-aspect-ratio flexible wings", Chinese Journal of Aeronautics, 22(4), pp. 355-363 (2009). DOI: $10.1016 / \mathrm{S} 1000-9361(08) 60111-9$

32. Hodges, D.H. "Geometrically exact, intrinsic theory for dynamics of curved and twisted anisotropic beams", AIA A Journal, 41(6), pp. 1131-1137 (2003). DOI: $10.2514 / 2.2054$

33. Eskandary, K., Dardel, M., Pashaei, M., et al. "Nonlinear aeroelastic analysis of high-aspect-ratio wings in low subsonic flow", Acta Astronautica, 70, pp. 6-22 (2012). DOI: $10.1016 /$ j.actaastro.2011.07.017

34. Badiei, D., Sadr, M., and Shams, S. "Static stall model in aeroelastic analysis of a flexible wing with geometrical nonlinearity", Journal of Aerospace Engineering, 27(2), pp. 378-389 (2012). DOI: 10.1061/(ASCE) AS.1943-5525.0000263

35. Dardel, M., Eskandary, K., Pashaei, M., et al. "The effect of angle of attack on limit cycle oscillations for high-aspect-ratio wings", Scientia Iranica, Transactions B, Mechanical Engineering, 21(1), p. 130 (2014). 
36. Xiao, Y.P., Yang, Y.R., and Li, P. "Limit-cycle oscillation of high-aspect-ratio wings", Applied Mechanics and Materials, 556-562, pp. 4329-4332, (2014). DOI: 10.4028/www.scientific.net/AMM.556-562.4329

37. Koohi, R., Shahverdi, H., and Haddadpour, H. "Nonlinear aeroelastic analysis of a composite wing by finite element method", Composite Structures, 113, pp. 118126 (2014).

38. Koohi, R., Shahverdi, H., and Haddadpour, H. "Modal and aeroelastic analysis of a high-aspect-ratio wing with large deflection capability", International Journal Advanced Design and Manufacturing Technology, 8(1), pp. $45-54$ (2015).

39. Yuan, K.A. and Friedmann, P.P. "Aeroelasticity and structural optimization of composite helicopter rotor blades with swept tips", National Aeronautics and Space Administration, Langley Research Center NASA $C R, 4665$ (1995).

40. Jung, Y.S., Yu, D.O., and Kwon, O.J. "Aeroelastic analysis of high-aspect-ratio wings using a coupled CFD-CSD method", Transactions of the Japan Society for Aeronautical and Space Sciences, 59(3), pp. 123133 (2016).

41. Bakhtiari-Nejad, F., Modarres, A., Dowell, E., et al. "Linear and nonlinear aeroelastic analysis of a high aspect ratio wing", In ASME 2017 International Design Engineering Technical Conferences and Computers and Information in Engineering Conference, Cleveland, Ohio (2017).

42. Hoseini, H. and Hodges, D.H. "Aeroelastic analysis of high aspect ratio wings using joined $3 \mathrm{D}$ finite elements and variational asymptotic beam models," 58th AIAA/ASCE/AHS/ASC Structures, Structural Dynamics, and Materials Conference, Grapevine, Texas (2017).

43. Farsadi, T., Rahmanian, M., and Kayran, A. "Geometrically nonlinear aeroelastic behavior of pretwisted composite wings modeled as thin walled beams", Journal of Fluids and Structures, 83, pp. 259-292 (2018).

44. Xu, Y., Cao, D., Lin, H., et al. "An alternative simulation approach for the ONERA aerodynamic model and its application in the nonlinear aeroelastic analysis of slender wings with pylon-store system", International Journal of Non-Linear Mechanics, 105, pp. 55-76 (2018).

45. Abbas, L., Chen, Q., Marzocca, P., et al. "Nonlinear aeroelastic investigations of store (s)-induced limit cycle oscillations", Proceedings of the Institution of Mechanical Engineers, Part G: Journal of Aerospace Engineering, 222(1), pp. 63-80 (2008). DOI: 10.1243/09544100JAERO241

46. Sina, S., Farsadi, T., and Haddadpour, H. "Aeroelastic stability and response of composite swept wings in subsonic flow using indicial aerodynamics", Journal of Vibration and Acoustics, 135(5), p. 051019 (2013). DOI: $10.1115 / 1.4023992$
47. Wright, J.R. and Cooper, J.E., Introduction to Aircraft Aeroelasticity and Loads, 20, pp. 76-80, John Wiley \& Sons, England (2008).

48. Rao, S.S., Vibration of Continuous Systems, pp. 333335, John Wiley \& Sons, United States of America (2007).

49. Fazelzadeh, S., Marzocca, P., Rashidi, E., et al. "Effects of rolling maneuver on divergence and flutter of aircraft wing store", Journal of Aircraft, 47(1), pp. 64-70 (2010). DOI: 10.2514/1.40463

50. Clark, R., Cox, D., Howard Jr, C., et al., A Modern Course in Aeroelasticity, 116, Springer Science \& Business Media (2006).

51. Goland, M. "The flutter of a uniform cantilever wing", Journal of Applied Mechanics-Transactions of the ASME, 12(4), pp. A197-A208 (1945).

52. Gern, F.H. and Librescu, L. "Effects of externally mounted stores on aeroelasticity of advanced swept cantilevered aircraft wings", Aerospace Science and Technology, 2(5), pp. 321-333 (1998). DOI: 10.1016/S1270-9638(98)80008-4

53. Patil, M., Hodges, D., and Cesnik, C. "Nonlinear aeroelastic analysis of aircraft with high-aspectratio wings", in Proceeding of 39th AIAA/ASME/ ASCE/AHS/ASC Structures, Structural Dynamics, and Materials Conference, AIAA paper 98-1955, Long Beach, CA (1998).

\section{Appendix}

Let $l, m$ and $n$ denote the numbers of in-plane $w$, outof-plane $v$, and torsional $\alpha$ modes, respectively. The elements of linear matrices are defined as follows:

$$
\begin{aligned}
& M_{i, j}=\int_{0}^{1} W_{i}(s) W_{j}(s) d s=\delta_{i j} \\
& 1 \leq i \leq l, \quad 1 \leq j \leq l, \\
& M_{l+i, l+j}=\int_{0}^{1} V_{i}(s) V_{j}(s) d s=\delta_{i j} \\
& 1 \leq i \leq m, \quad 1 \leq j \leq m, \\
& M_{l+i, l+m+j}=-e \int_{0}^{1} V_{i}(s) A_{j}(s) d s \\
& 1 \leq i \leq m, \quad 1 \leq j \leq n, \\
& M_{l+m+i, l+j}=-e \int_{0}^{1} A_{i}(s) V_{j}(s) d s \\
& 1 \leq i \leq n, \quad 1 \leq j \leq m,
\end{aligned}
$$


$M_{l+m+i, l+m+j}=-j_{1} \int_{0}^{1} A_{i}(s) A_{j}(s) d s=-j_{1} \delta_{i j}$

$1 \leq i \leq n, \quad 1 \leq j \leq n$,

$K_{i, j}=\beta_{z} \int_{0}^{1} W_{i}(s) W_{j}^{i v}(s) d s=\beta_{z} z_{j}^{4} \delta_{i j}$

$1 \leq i \leq l, \quad 1 \leq j \leq l$,

$K_{l+i, l+j}=\beta_{z} \int_{0}^{1} V_{i}(s) V_{j}^{i v}(s) d s=\beta_{z} z_{j}^{4} \delta_{i j}$

$1 \leq i \leq m, \quad 1 \leq j \leq m$,

$K_{l+m+i, l+m+j}=-\beta_{y} \int_{0}^{1} A_{i}(s) A_{j}^{\prime \prime}(s) d s=\beta_{y} \alpha_{j}^{2} \delta_{i j}$

$1 \leq i \leq n, \quad 1 \leq j \leq n$,

$C_{i, j}=c_{w_{i}} \int_{0}^{1} W_{i}(s) W_{j}(s) d s=c_{w_{i}} \delta_{i j}$

$1 \leq i \leq l, \quad 1 \leq j \leq l$

$C_{l+i, l+j}=c_{v_{i}} \int_{0}^{1} V_{i}(s) V_{j}(s) d s=c_{v_{i}} \delta_{i j}$

$1 \leq i \leq m, \quad 1 \leq j \leq m$,

$C_{l+m+i, l+m+j}=c_{\alpha_{i}} \int_{0}^{1} A_{i}(s) A_{j}(s) d s=c_{\alpha_{i}} \delta_{i j}$

$1 \leq i \leq n, \quad 1 \leq j \leq n$.

Note that the linear matrices are squares on the order of $l+m+n$ and the subscripts represent the row and column of each element. Similarly, the elements of nonlinear matrices may be determined as:

$$
\begin{aligned}
M_{i, j}^{n l}= & j_{2} \int_{0}^{1} W_{i} W^{\prime \prime}{ }_{j} d s-\sum_{p=1}^{l} \sum_{q=1}^{l} \int_{0}^{1} W_{i} \\
& \left(W^{\prime}{ }_{p} \int_{1}^{s} \int_{0}^{s} W^{\prime}{ }_{j} W^{\prime}{ }_{q} d s d s\right)^{\prime} d s w_{p} \dot{w}_{q} \\
& -e_{z} \sum_{p=1}^{l} \int_{0}^{1} W_{i}\left[\left(W^{\prime}{ }_{p} \int_{1}^{s} W_{j}{ }_{j} d s+W_{p}{ }_{p} W_{j}\right)^{\prime}\right.
\end{aligned}
$$

$$
\begin{aligned}
& \left.+2 W^{\prime}{ }_{j} W^{\prime}{ }_{p}\right] d s w_{p} \\
& -2 e_{z} \sum_{p=1}^{m} \sum_{k=1}^{n} \int_{0}^{1} W_{i} A_{k} \int_{0}^{s} V^{\prime \prime}{ }_{p} W^{\prime}{ }_{j} d s d s v_{p} \alpha_{k}
\end{aligned}
$$

$1 \leq i \leq n, \quad 1 \leq j \leq n$

$$
\begin{aligned}
M_{i, l+j}^{n l}= & -\sum_{q=1}^{l} \sum_{p=1}^{m} \int_{0}^{1} W_{i} \\
& \left(W_{q}{ }^{\prime} \int_{1}^{s} \int_{0}^{s} V_{j}{ }^{\prime} V_{p}{ }^{\prime} d s d s\right)^{\prime} d s w_{q} v_{p} \\
& -e_{z} \sum_{p=1}^{m} \int_{0}^{1} W_{i}\left[\left(V_{p}{ }^{\prime} V_{j}{ }^{\prime}\right)^{\prime}-V_{j}{ }^{\prime} V_{p}{ }^{\prime}\right] d s v_{p} \\
& +e_{z} \sum_{p=1}^{l} \sum_{k=1}^{n} \int_{0}^{1} W_{i}\left[\left(W_{p}{ }^{s}{ }_{1} V_{j}{ }^{\prime} A_{k} d s\right)^{\prime}\right. \\
& \left.-A_{k} \int_{0}^{s} V_{j}{ }^{\prime \prime} W_{p}{ }^{\prime} d s\right] d s w_{p} \alpha_{k}
\end{aligned}
$$

$1 \leq i \leq l, \quad 1 \leq j \leq m$

$$
\begin{aligned}
& M_{i, l+m+j}^{n l}=e_{z} \sum_{p=1}^{m} \sum_{q=1}^{l} \int_{0}^{1} W_{i} \\
& {\left[\left(W_{q}^{\prime} \int_{1}^{s} V_{p}^{\prime} A_{j} d s\right)^{\prime}-A_{j} \int_{0}^{s} V_{p}^{\prime \prime} W_{q}^{\prime} d s\right] } \\
& d s v_{p} w_{q}+e_{z} \sum_{p=1}^{n} \int_{0}^{1} W_{i} A_{j} A_{p} d s \alpha_{p}
\end{aligned}
$$

$1 \leq i \leq l, \quad 1 \leq j \leq n$,

$$
\begin{aligned}
M_{l+i, j}^{n l} & =-\sum_{p=1}^{m} \sum_{q=1}^{l} \int_{0}^{1} V_{i}\left(V_{p}{ }^{\prime} \int_{1}^{s} \int_{0}^{s} W_{j}{ }^{\prime} W_{q}{ }^{\prime} d s d s\right)^{\prime} d s v_{p} w_{q} \\
& -e_{z} \sum_{k=1}^{n} \sum_{p=1}^{l} \int_{0}^{1} V_{i}\left[A_{k}\left(\int_{0}^{s} W_{j}{ }^{\prime} W_{p}{ }^{\prime} d s-W_{p}^{\prime} W_{j}\right)\right]^{\prime} \\
& d s \alpha_{k} w_{p}-e_{z} \sum_{p=1}^{m} \int_{0}^{1} V_{i}\left[\left(V_{p}^{\prime} \int_{1}^{s} W_{j}^{\prime} d s\right)^{\prime}\right.
\end{aligned}
$$




$$
\left.-\int_{0}^{s} V_{p}^{\prime} W_{j}^{\prime \prime} d s\right] d s v_{p}
$$

$1 \leq i \leq m, \quad 1 \leq j \leq l$,

$$
\begin{aligned}
M_{l+i, l+j}^{n l} & =j_{3} \int_{0}^{1} V_{i} V_{j}{ }^{\prime \prime} d s+e_{z} \sum_{p=1}^{l} \int_{0}^{1} V_{i} \int_{0}^{s} V_{j}{ }^{\prime} W_{p}{ }^{\prime \prime} d s d s w_{p} \\
& -\sum_{p=1}^{m} \sum_{q=1}^{m} \int_{0}^{1} V_{i}\left(V_{p}{ }^{s} \int_{1}^{s} \int_{0}^{s} V_{j}{ }^{\prime} V_{q}{ }^{\prime} d s d s\right)^{\prime} d s v_{p} v_{q} \\
& -e_{z} \sum_{p=1}^{m} \sum_{k=1}^{n} \int_{0}^{1} V_{i}\left[\left(A_{k} \int_{0}^{s} V_{j}{ }^{\prime} V_{p}{ }^{\prime} d s-A_{k} V_{p}{ }^{\prime} V_{j}\right.\right. \\
& \left.\left.-V_{p}{ }^{\prime} \int_{1}^{s} V_{j}{ }^{\prime} A_{k} d s\right)^{\prime}+V_{j}{ }^{\prime} V_{p}{ }^{\prime} A_{k}\right] d s v_{p} \alpha_{k}
\end{aligned}
$$

$1 \leq i \leq m, \quad 1 \leq j \leq m$

$$
\begin{gathered}
M_{l+i, l+m+j}^{n l}=\frac{e_{z}}{2} \sum_{p=1}^{m} \sum_{q=1}^{m} \int_{0}^{1} V_{i}\left[2\left(V_{p}^{\prime} \int_{1}^{s} V_{q}^{\prime} A_{j} d s\right)^{\prime}\right. \\
\left.-V_{p}^{\prime} V_{q}^{\prime} A_{j}\right] d s v_{p} v_{q} \\
-\frac{1}{2} e_{z} \sum_{p=1}^{n} \sum_{q=1}^{n} \int_{0}^{1} V_{i} A_{j} A_{p} A_{q} d s \alpha_{p} \alpha_{q}
\end{gathered}
$$

$1 \leq i \leq m, \quad 1 \leq j \leq n$,

$$
\begin{aligned}
& M_{l+m+i, j}^{n l}=j_{1} \sum_{p=1}^{m} \int_{0}^{1} A_{i} \int_{0}^{s} V_{p}{ }^{\prime} W_{j}{ }^{\prime} \\
& d s d s v_{p}+e_{z} \sum_{p=1}^{m} \sum_{q=1}^{l} \int_{0}^{1} A_{i}\left[V_{p}{ }^{\prime} \int_{0}^{s} W_{j}{ }^{\prime} W_{q}{ }^{\prime} d s\right. \\
& \left.-W_{j} \int_{0}^{s} V_{p}{ }^{\prime} W_{q}{ }^{\prime} d s\right] d s v_{p} w_{q} \\
& +e_{z} \sum_{k=1}^{n} \int_{0}^{1} A_{i} A_{k} W_{j} d s \alpha_{k}
\end{aligned}
$$

$1 \leq i \leq n, \quad 1 \leq j \leq l$,

$$
M_{l+m+i, l+j}^{n l}=j_{1} \sum_{p=1}^{l} \int_{0}^{1} A_{i}\left[\int_{0}^{s} V_{j}{ }^{\prime \prime} W_{p}{ }^{\prime} d s-V_{j}{ }^{\prime} W_{p}{ }^{\prime}\right]
$$$$
d s w_{p} \ddot{v}_{j}+\frac{1}{2} e_{z}\left\{\sum_{p=1}^{m} \sum_{q=1}^{m} \int_{0}^{1} A_{i} V_{p}^{\prime}\right.
$$$$
\left[2 \int_{0}^{s} V_{j}^{\prime} V_{q}^{\prime} d s-V_{q}^{\prime} V_{j}\right] d s v_{p} v_{q} \ddot{v}_{j}
$$$$
\left.-\sum_{k=1}^{n} \sum_{p=1}^{n} \int_{0}^{1} A_{i} A_{k} A_{p} V_{j} d s \alpha_{k} \alpha_{p}\right\}
$$

$1 \leq i \leq n, \quad 1 \leq j \leq m$,

$$
\begin{gathered}
C_{i, j}^{n l}=-\sum_{q=1}^{l} \sum_{p=1}^{l} \int_{0}^{1} W_{i}\left(W_{q}{ }^{\prime} \int_{1}^{s} \int_{0}^{s} W_{p}{ }^{\prime} W_{j}{ }^{\prime} d s d s\right)^{\prime} \\
v d s w_{q} \dot{w}_{p}+2 e_{z} \sum_{p=1}^{l} \int_{0}^{1} W_{i} W_{j}{ }^{\prime} W_{p}{ }^{\prime} d s \dot{w}_{p} \\
\quad-2 e_{z} \sum_{p=1}^{m} \sum_{k=1}^{n} \int_{0}^{1} W_{i} A_{k} \int_{0}^{s} V_{p}{ }^{\prime \prime} W_{j}{ }^{\prime} d s d s \\
\left(\dot{\alpha}_{k} v_{p}+\alpha_{k} \dot{v}_{p}\right) \\
1 \leq i \leq l, \quad 1 \leq l, \\
C_{i, l+j}^{n l}=-\sum_{q=1}^{l} \sum_{p=1}^{m} \int_{0}^{1} W_{i}\left(W_{q} \int_{1}^{s} \int_{0}^{s} V_{j}{ }^{\prime} V_{p}{ }^{\prime} d s d s\right)^{\prime}
\end{gathered}
$$$$
d s w_{q} \dot{v}_{p}+e_{z} \sum_{p=1}^{m} \int_{0}^{1} W_{i} V_{j}^{\prime} V_{p}^{\prime} d s \dot{v}_{p}
$$$$
+2 e_{z} \sum_{p=1}^{l} \sum_{k=1}^{n} \int_{0}^{1} W_{i}\left[\left(W_{p}^{\prime} \int_{1}^{s} V_{j}^{\prime} A_{k}\right)^{\prime}\right.
$$$$
\left.-A_{k} \int_{0}^{s} V_{j}^{\prime \prime} W_{p}^{\prime} d s\right] d s w_{p} \dot{\alpha}_{k}
$$

$1 \leq i \leq l, \quad 1 \leq j \leq m$,

$C_{i, m+l+j}^{n l}=e_{z} \sum_{k=1}^{n} \int_{0}^{1} W_{i} A_{k} A_{j} d s \dot{\alpha}_{k} \dot{\alpha}_{j}$

$1 \leq i \leq l, \quad 1 \leq j \leq n$, 


$$
\begin{aligned}
C_{l+i, j}^{n l}= & -\sum_{p=1}^{m} \sum_{q=1}^{l} \int_{0}^{1} V_{i}\left(V_{p}{ }^{\prime} \int_{1}^{s} \int_{0}^{s} W_{j}{ }^{\prime} W_{q}{ }^{\prime} d s d s\right)^{\prime} \\
& d s v_{p} \dot{w}_{q}-e_{z} \sum_{k=1}^{n} \sum_{p=1}^{l} \int_{0}^{1} V_{i}\left(A_{k} \int_{0}^{s} W_{j}{ }^{\prime} W_{q}{ }^{\prime} d s\right)^{\prime} \\
& d s \alpha_{k} \dot{w}_{p}+2 e_{z} \sum_{p=1}^{m} \int_{0}^{1} V_{i} \int_{0}^{s} V_{p}{ }^{\prime} W_{j}{ }^{\prime \prime} d s d s \dot{v}_{p}
\end{aligned}
$$

$1 \leq i \leq m, \quad 1 \leq j \leq l$,

$$
\begin{aligned}
C_{l+i, l+j}^{n l}= & -\sum_{p=1}^{m} \sum_{q=1}^{m} \int_{0}^{1} V_{i}\left(V_{p}{ }^{\prime} \int_{1}^{s} \int_{0}^{s} V_{j}^{\prime} V_{q}^{\prime} d s d s\right)^{\prime} \\
& d s v_{p} \dot{v}_{q}-e_{z} \sum_{p=1}^{m} \sum_{k=1}^{n} \int_{0}^{1} V_{i}\left[\left(A_{k} \int_{0}^{s} V_{j}^{\prime} V_{p}^{\prime} d s\right)^{\prime}\right. \\
& \left.+V_{j}^{\prime} V_{p}^{\prime} A_{k}\right] d s \dot{v}_{p} \alpha_{k}+2 e_{z} \sum_{p=1}^{m} \sum_{k=1}^{n} \int_{0}^{1} V_{i} \\
& {\left[\left(V_{p}^{\prime} \int_{1}^{s} V_{j}^{\prime} A_{k} d s\right)^{\prime}-V_{j}^{\prime} V_{p}^{\prime} A_{k}\right] d s v_{p} \dot{\alpha}_{k} }
\end{aligned}
$$

$1 \leq i \leq m, \quad 1 \leq j \leq m$,

$C_{l+i, l+m+j}^{n l}=-e_{z} \sum_{p=1}^{n} \sum_{q=1}^{n} \int_{0}^{1} V_{i} A_{j} A_{p} A_{q} d s \dot{\alpha}_{p} \alpha_{q}$

$1 \leq i \leq m, \quad 1 \leq j \leq n$,

$$
\begin{aligned}
C_{l+m+i, j}^{n l} & =\sum_{p=1}^{m} \int_{0}^{1} A_{i}\left[j_{1}\left(2 \int_{0}^{s} V_{p}{ }^{\prime \prime} W_{j}{ }^{\prime} d s-V_{p}{ }^{\prime} W_{j}{ }^{\prime}\right)\right. \\
& \left.-\left(j_{2}-j_{3}\right) V_{p}{ }^{\prime} W_{j}{ }^{\prime}\right] d s \dot{v}_{p} \\
& -\left(j_{2}-j_{3}\right) \sum_{p=1}^{l} \sum_{k=1}^{n} \int_{0}^{1} A_{i} W_{j}{ }^{\prime} W_{p}{ }^{\prime} A_{k} d s \dot{w}_{p} \alpha_{k} \\
& +e_{z} \sum_{p=1}^{m} \sum_{q=1}^{l} \int_{0}^{1} A_{i} V_{p}{ }^{\prime} \int_{0}^{s} W_{j}{ }^{\prime} W_{q}{ }^{\prime} d s d s v_{p} \dot{w}_{q}
\end{aligned}
$$

$1 \leq i \leq n, \quad 1 \leq j \leq l$,

$$
\begin{gathered}
C_{l+m+i, l+j}^{n l}=\left(j_{2}-j_{3}\right) \sum_{p=1}^{m} \sum_{k=1}^{n} \int_{0}^{1} A_{i} V_{j}{ }^{\prime} V_{p} A_{k} d s \dot{v}_{p} \alpha_{k} \\
+e_{z} \sum_{p=1}^{m} \sum_{q=1}^{m} \int_{0}^{1} A_{i} V_{p}^{\prime} \int_{0}^{s} V_{j}{ }^{\prime} V_{q}{ }^{\prime} d s d s v_{p} \dot{v}_{q} \\
1 \leq i \leq n, \quad 1 \leq j \leq m, \\
K_{i, j}^{n l}=-\beta_{z} \sum_{p=1}^{l} \sum_{q=1}^{l} \int_{0}^{1} W_{i}\left[W_{j}{ }^{\prime}\left(W_{p}{ }^{\prime} W_{q}{ }^{\prime \prime}\right)^{\prime}\right]^{\prime} d s w_{p} w_{q} \\
+\left(\beta_{z}-1\right) \sum_{k=1}^{n} \sum_{p=1}^{n} \int_{0}^{1} W_{i}\left(W_{j}{ }^{\prime \prime} A_{k} A_{p}\right)^{\prime \prime} d s \alpha_{k} \alpha_{p} \\
-\sum_{p=1}^{m} \sum_{q=1}^{m} \int_{0}^{1} W_{i}\left[\beta_{z} W_{j}{ }^{\prime}\left(V_{q}{ }^{\prime} V_{p}{ }^{\prime \prime}\right)^{\prime}\right. \\
\left.-\left(\beta_{z}-1\right) V_{q}^{\prime \prime \prime} \int_{0}^{s} V_{p}{ }^{\prime} W_{j}{ }^{\prime \prime} d s\right]^{\prime} d s v_{p} v_{q}
\end{gathered}
$$

$1 \leq i \leq l, \quad 1 \leq j \leq l$,

$K_{i, l+j}^{n l}=\sum_{k=1}^{n} \int_{0}^{1} W_{i}\left[\beta_{y}\left(A_{k}{ }^{\prime} V_{j}{ }^{\prime \prime}\right)^{\prime}+\left(\beta_{z}-1\right)\left(V_{j}{ }^{\prime \prime} A_{k}\right)^{\prime \prime}\right]$

$$
d s \alpha_{k} \quad 1 \leq i \leq l, \quad 1 \leq j \leq m,
$$

$$
K_{l+i, j}^{n l}=-\sum_{k=1}^{n} \int_{0}^{1} V_{i}\left[\beta_{y}\left(A W_{j}^{\prime \prime}\right)^{\prime}\right.
$$

$$
\begin{aligned}
& \left.-\left(\beta_{z}-1\right)\left(W_{j}^{\prime \prime} A_{k}\right)^{\prime \prime}\right] d s \alpha_{k} \\
& -\sum_{p=1}^{m} \sum_{q=1}^{l} \int_{0}^{1} V_{i}\left[V_{p}{ }^{\prime}\left(W_{j}{ }^{\prime} W_{q}{ }^{\prime \prime}\right)^{\prime}\right. \\
& \left.+\left(\beta_{z}-1\right)\left(W_{j}^{\prime \prime \prime} \int_{0}^{s} V_{p}{ }^{\prime \prime} W_{q}{ }^{\prime} d s\right)\right]^{\prime} d s v_{p} w_{q}
\end{aligned}
$$

$1 \leq i \leq m, \quad 1 \leq j \leq l$,

$$
K_{l+i, l+j}^{n l}=-\sum_{p=1}^{m} \sum_{q=1}^{m} \int_{0}^{1} V_{i}\left[V_{j}{ }^{\prime}\left(V_{p}{ }^{\prime} V_{q}{ }^{\prime \prime}\right)^{\prime}\right]^{\prime} d s v_{p} v_{q}
$$




$$
-\left(\beta_{z}-1\right) \sum_{k=1}^{n} \sum_{p=1}^{n} \int_{0}^{1} V_{i}\left(V_{j}^{\prime \prime} A_{k} A_{p}\right)^{\prime \prime} d s \alpha_{k} \alpha_{p}
$$

$1 \leq i \leq m, \quad 1 \leq j \leq m$,

$$
\begin{gathered}
K_{l+m+i, j}^{n l}=-\left(1-\beta_{z}\right)\left[\sum_{p=1}^{l} \sum_{k=1}^{n} \int_{0}^{1} A_{i} W_{j}^{\prime \prime} W_{p}^{\prime \prime} A_{k}\right. \\
\left.d s w_{p} \alpha_{k}+\sum_{p=1}^{m} \int_{0}^{1} A_{i} V_{P}{ }^{\prime \prime} W_{j}{ }^{\prime \prime} d s v_{p}\right]
\end{gathered}
$$

$1 \leq i \leq n, \quad 1 \leq j \leq l$,

$K_{l+m+i, l+j}^{n l}=\left(1-\beta_{z}\right) \sum_{p=1}^{m} \sum_{k=1}^{n} \int_{0}^{1} A_{i} V_{j}^{\prime \prime} V_{p}{ }^{\prime \prime} A_{k} d s v_{p} \alpha_{k}$

$1 \leq i \leq n, \quad 1 \leq j \leq m$.

To obtain the elements of aerodynamic mass, damping, and stiffness matrices, the following integral statements can be evaluated:

$$
M_{l+i, l+j}^{a}=-\mu \int_{0}^{1} V_{i}(s) V_{j}(s) d s
$$

$1 \leq i \leq n, \quad 1 \leq j \leq m$,

$$
M_{l+i, m+l+j}^{a}=\mu a b \int_{0}^{1} V_{i}(s) A_{j}(s) d s
$$

$1 \leq i \leq m, \quad 1 \leq j \leq n$,

$M_{l+m+i, l+j}^{a}=\mu a b \int_{0}^{1} A_{i}(s) V_{j}(s)$

$1 \leq i \leq n, \quad 1 \leq j \leq m$,

$M_{l+m+i, l+m+j}^{a}=-\mu b^{2}\left(\frac{1}{8}+a^{2}\right) \int_{0}^{1} A_{m}(s) A_{k}(s)$

$1 \leq i \leq n, \quad 1 \leq j \leq n$,

$C_{l+i, l+j}^{a}=-c_{a} \phi_{c \alpha}(0) \int_{0}^{1} V_{i}(s) V_{j}(s) d s$

$1 \leq i \leq m, \quad 1 \leq j \leq m$,

$C_{l+i, l+m+j}^{a}=-\left[\mu U+2 c_{a} b \phi_{c q}(0)\right] \int_{0}^{1} V_{i}(s) A_{j}(s) d s$
$1 \leq i \leq m, \quad 1 \leq j \leq n$,

$C_{l+m+i, l+j}^{a}=2 b c_{a} \phi_{c m}(0) \int_{0}^{1} A_{i}(s) V_{j}(s) d s$

$1 \leq i \leq n, \quad 1 \leq j \leq m$,

$C_{l+m+i, l+m+j}^{a}=\left[4 b^{2} c_{a} \phi_{c m q}(0)+\mu a b U\right]$

$$
\int_{0}^{1} A_{i}(s) A_{j}(s) d s
$$

$1 \leq i \leq n, \quad 1 \leq j \leq n$

$K_{l+i, l+j}^{a}=-c_{a} \dot{\phi}_{c \alpha}(0) \int_{0}^{1} V_{i}(s) V_{j}(s) d s$

$1 \leq i \leq m, \quad 1 \leq j \leq m$

$K_{l+i, l+m+j}^{a}=-c_{a}\left[U \phi_{c \alpha}(0)+2 b \dot{\phi}_{c q}(0)\right]$

$$
\int_{0}^{1} V_{i}(s) A_{j}(s) d s
$$

$1 \leq i \leq m, \quad 1 \leq j \leq n$,

$K_{l+m+i, l+j}^{a}=2 b c_{a} \dot{\phi}_{c m}(0) \int_{0}^{1} A_{i}(s) V_{j}(s) d s$

$1 \leq i \leq n, \quad 1 \leq j \leq m$,

$$
\begin{aligned}
K_{l+m+i, l+m+j}^{a}= & 2 b c_{a}\left[U \phi_{c m}(0)+2 b \dot{\phi}_{c m q}(0)\right] \\
& \int_{0}^{1} A_{i}(s) A_{j}(s) d s
\end{aligned}
$$

$1 \leq i \leq n, \quad 1 \leq j \leq n$.

Finally, the initial condition matrix [IC] can also be determined as:

$$
\begin{aligned}
& I C_{l+i, l+j}=c_{a} \dot{\phi}_{c \alpha} \int_{0}^{1} V_{i}(s) V_{j}(s) d s \\
& 1 \leq i \leq m, \quad 1 \leq j \leq m, \\
& I C_{l+i, l+m+j}=2 c_{a} b \dot{\phi}_{c q} \int_{0}^{1} V_{i}(s) A_{j}(s) d s \\
& 1 \leq i \leq m, \quad 1 \leq j \leq n,
\end{aligned}
$$




$$
\begin{aligned}
& I C_{l+m+i, l+j}=-2 b c_{a} \dot{\phi}_{c m} \int_{0}^{1} A_{i}(s) V_{j}(s) d s \\
& 1 \leq i \leq n, \quad 1 \leq j \leq m, \\
& I C_{l+m+i, l+m+j}=-4 b^{2} c_{a} \dot{\phi}_{c m q} \int_{0}^{1} A_{i}(s) A_{j}(s) d s
\end{aligned}
$$

$1 \leq i \leq n, \quad 1 \leq j \leq n$

It should be noted that all the elements not mentioned in the above matrices are zero.

\section{Biographies}

Mahdi Nejati received his BSc degree in Mechanical Engineering from Islamic Azad University, Tehran, Iran, in 1998 and his MSc degree in Aerospace Engineering from Malek-Ashtar University of Technology in 2010, Tehran, Iran. He is currently studying for his $\mathrm{PhD}$ degree in Aerospace Engineering at Malek-Ashtar University of Technology, Tehran, Iran. His research interests are in the field of aeroelasticity, structural dynamics, and nonlinear vibrations.

Saeed Shokrollahi received his BSc degree in Mechanical Engineering from Shiraz University, Shiraz, Iran, in 1994; his MSc degree in Mechanical Engineering from University of Tehran in 1997; and his
PhD degree in Mechanical Engineering from Amirkabir University of Technology, Tehran, Iran, in 2004. $\mathrm{He}$ is currently an Associate Professor of Mechanical Engineering in the Aerospace Engineering Department of Malek-Ashtar University of Technology, Tehran, Iran. His research interests are in the field of aeroelasticity, including the study of dynamic interaction between aerodynamic flow and elastic structures such as aircraft wings during high-speed flight. He has also conducted research on experimental modal analysis, nonlinear identification, structural dynamics, and unsteady aerodynamics. He has supervised more than $30 \mathrm{MS}$ theses and three $\mathrm{PhD}$ dissertations since 2004.

Shahrokh Shams is currently an Assistant Professor in the Department of Aerospace Engineering, Faculty of New Sciences and Technologies, University of Tehran, Tehran, Iran. He obtained his BS, MS, and $\mathrm{PhD}$ degrees from Amirkabir University of Technology up to 2008. His research interests are in the areas of vibration, aeroelasticity, and composite material analysis.

Ramin Torkaman received his BSc degree in Mechanical Engineering from Islamic Azad University, Kashan, Iran, in 2011 and his MSc degree in Aerospace Engineering from Malek-Ashtar University of Technology in 2015, Tehran, Iran. He is interested in nonlinear vibration and nonlinear aeroelasticity. 